(C) Copyright 2002 IEEE. IEEE Transactions on Vehicular Technology November 2002, vol.51, no.6, pp.1279-1293

Personal use of this material is permitted. However, permission to reprint/republish this material for advertising or promotional purposes or for creating new collective works for resale or redistribution to servers or lists, or to reuse any copyrighted component of this work in other works must be obtained from the IEEE. 


\title{
A Real-Time DOA-Based Smart Antenna Processor
}

\author{
Alexander Kuchar, Member, IEEE, Michael Tangemann, Member, IEEE, and Ernst Bonek, Senior Member, IEEE
}

\begin{abstract}
We designed, built, and tested a real-time processor for a direction-of-arrival-based smart antenna GSM 1800 base station with eight half-wavelength spaced antenna elements. Its processing steps include direction-of-arrival (DOA) estimation, user identification, tracking, beamforming, and signal reconstruction. We demonstrate that the accuracy of DOA estimation is not of primary concern, but the robustness is. This can be assessed by a new parameter, the estimation range. Tracking of reliable user DOAs only, increases the robustness against interference. Our tracking concept is compatible with frequency hopping. We quantify the benefits of smart antennas by the statistics of the gain in carrier-tointerference ratio $(\mathrm{C} / \mathrm{I})$ and in signal-to-noise ratio (SNR), both measured at the 90 or $99 \%$ levels with actual transmitted data. In an urban environment with large angular spread and overlap of user and interferer signals, the $\mathrm{C} / \mathrm{I}$ gain is as high as $18 \mathrm{~dB}$. Interferer nulling increases the $\mathrm{C} / \mathrm{I}$ gain only slightly, but enhances robustness against angular spread, particularly if broad nulls are applied. Separating SNR gain in its contributions due to beamforming and diversity gives valuable insight into the way of operation. In uplink, the processor can exploit angular diversity. The entire suite of processing steps is done within less than $1 \mathrm{~ms}$, demonstrating that sophisticated DOA-based smart antenna processing is feasible in real time. Our solution does not require any change in the GSM standard.
\end{abstract}

Index Terms-Angular diversity, carrier-to-interference ratio (C/I) improvement, component angular spread, direction-of-arrival estimation, smart antenna, wireless communications.

\section{INTRODUCTION}

$\mathbf{S}$ MART antennas exploit the spatial dimension. They are strong candidates to overcome the capacity limits of second-generation systems, as well as to assist coexistence of high and low data rate users in third-generation systems. Smart antennas affect the link budget positively, meaning that they can enhance coverage. Selecting one strong multipath out of many will reduce frequency selective fading and increase maximum possible data rate.

Smart antenna technology is on the brink of commercial realization. Although there is enough room for pioneering work of theoretical nature, at this stage another issue is of importance: Smart antenna technology must be proven practically.

Implementing smart antenna technology for real-time operation is a challenge for hardware as well as for array processing.

Manuscript received August 22, 2000; revised August 30, 2001. This paper was presented in part at VTC'99 in Houston, TX, and ICC'99, Vancouver, Canada.

A. Kuchar is with the Forschungszentrum Telekommunikation Wien (FTW), A-1040 Wien, Vienna, Austria (e-mail: kuchar@ftw.at).

M. Tangemann is with Alcatel SEL Stuttgart, Germany (e-mail: Michael.Tangemann@Alcatel.de).

E. Bonek is with the Forschungszentrum Telekommunikation Wien (FTW), A-1040 Wien, Vienna, Austria and also with the Institut für Nachrichtentechnik und Hochfrequenztechnik, Technische Universität Wien, Vienna, Austria (e-mail: Ernst.Bonek@tuwien.ac.at).

Digital Object Identifier 10.1109/TVT.2002.801737
Several approaches have been studied to introduce smart antenna technology into GSM [6], [2], [17], IS-136 [24], [8], and third generation systems [16], [1]. Most of these schemes either include uplink processing only, or apply separate algorithmic solutions in up- and downlink.

In this work both uplink and downlink are treated in a single homogenous directions-of-arrival (DOA)-based approach. In Section II we present the developed GSM1800 smart antenna base station [13], [14]. Its heart is the real-time Adaptive Antenna Array Processor $\mathrm{A}^{3} \mathrm{P}$ (Section III). It works within the GSM standard and is compatible with frequency hopping. In a first stage the smart antenna is designed to suppress co-channel interference, i.e., we apply Spatial Filtering for Interference Reduction (SFIR) [21]. In Section IV we will define the quantities with which we assess $A^{3} P$ in mobile radio channels, and find means to separate $C / I$, diversity, and beamforming gains. To evaluate the complete system we performed an extensive measurement campaign with actual transmitted data. The measurement modeling will be described in Section V. In Section VI we will set the requirements on the angular resolution of the DOA estimators. Our assessment of the DOA estimators will indicate that the estimation accuracy of the estimator is not of primary concern, but the robustness is. We will define a new measure to quantify the robustness. In this assessment, Monte Carlo simulations will complement the measurements. We will demonstrate the importance of the tracking concept of $A^{3} P$ in Section VII and highlight its key properties. The question whether broad nulls are advantageous will be tackled in Section VIII. Based on the overall performance we will discuss the results and general rules for the various processing modules in Section IX.

In the Appendix we will define a component angular spread that allows better judgement of channel situations in context with DOA-based smart antennas than the angular spread per se.

\section{SYSTEM CONCEPT}

Many of today's mobile communication systems use frequency division duplex (FDD) for up- and downlink transmission, in which the small-scale (Rayleigh) fading is uncorrelated. Reusing the uplink weights for downlink beamforming can lead to erroneous results. But channel parameters like the directions-of-arrival (DOAs) and the path loss are the same in uplink and in downlink.

Thus we base our array processing on DOA estimation in uplink. The estimated DOAs are tracked in uplink and in downlink separately to form antenna patterns that suppress co-channel interference. DOA-based beamforming relies on the assumption of almost-plane incident waves. Still, there exist uncorrelated antenna signal portions, which means that a diversity gain is also possible. In fact, the effects of fading can be mitigated by 


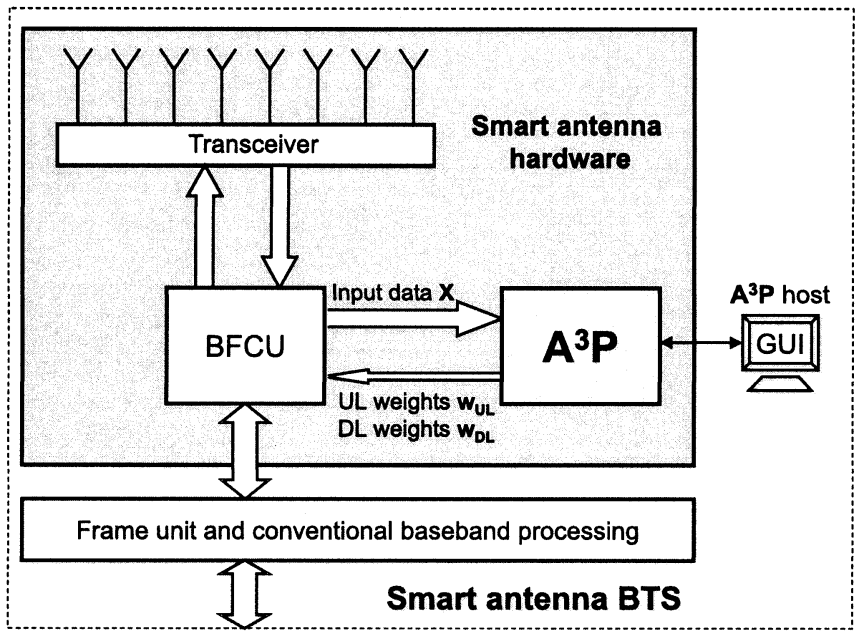

Fig. 1. Hardware concept of the GSM1800 smart antenna base transceiver station (BTS). The BTS consists of a conventional BTS frame unit, and smart antenna relevant hardware components, including the $A^{3} P$ and a beamforming control unit (BFCU). A graphical user interface (GUI), on the $A^{3} P$ host, controls the operation of the $A^{3} P$

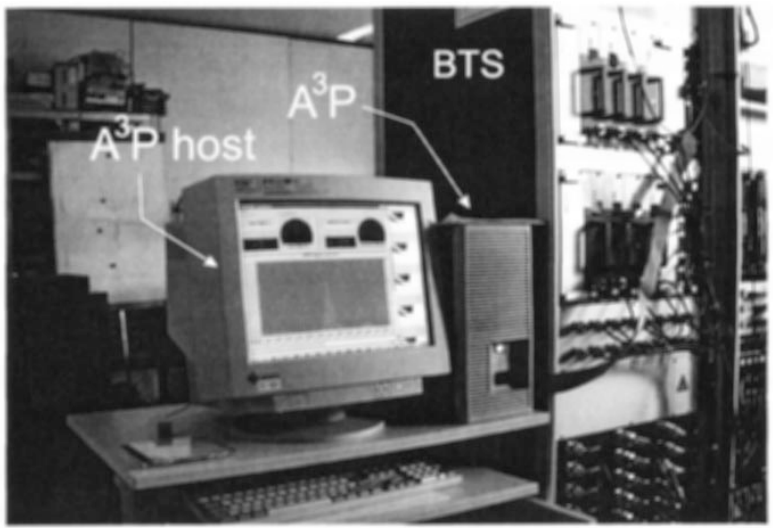

Fig. 2. $A^{3} P$, the $A^{3} P$ host and the base transceiver station (BTS).

exploiting angular diversity in the uplink. The testbed also provides angular diversity, but this is not covered by the results presented in this paper. In this paper, we focus on measurements demonstrating interference reduction. In noise limited environments, a diversity gain was achieved [12].

We designed our smart-antenna processor, called Adaptive Antenna Array Processor $A^{3} \mathrm{P}$, around an existing GSM 1800 base transceiver station (BTS). Concerning hardware, the BTS uses one and the same uniform linear eight-element antenna array with a half-wavelength inter-element spacing in up- and downlink (see Figs. 1 and 2). In uplink, all eight branches are downconverted and sampled in $I$ - and $Q$-signal paths to allow full adaptation of the beamforming weights. The Adaptive Antenna Array Processor $A^{3} P$ uses the sampled and calibrated input data received during a time slot in the uplink to calculate the antenna weights for up- and downlink beamforming. It is implemented on a single general purpose processor (DEC Alpha $500 \mathrm{MHz}$ ), which offers a flexible programming environment while still providing enough computing power to allow a real-time pattern adaptation in every GSM frame (4.6 ms). All algorithms are run-time optimized in $C$ and take about $1 \mathrm{~ms}$ for a complete weight calculation. The beamforming control unit (BFCU) performs the physical beamforming (multiplication of the received signals with the complex weight vectors) and controls all system components. Simple algorithms (e.g., switched beam) are incorporated in the BFCU for real-time comparison with the complex algorithms of $A^{3} P$.

In principle, our DOA-based smart antenna consists of the following processing modules: A DOA estimation, a DOA identification to link the DOAs with the user or interferer, a tracker, and a final beamformer.

A careful selection of a DOA estimator that suits the requirements of the mobile radio channel is important. Concerning high-resolution DOA estimation, we will investigate whether angular resolution in the subdegree range is required. Our goal is to relax requirements on angle estimation to reduce the complexity of the system. ${ }^{1}$ The suppression of interferers by placing nulls requires the knowledge of the interferer DOAs. We will take a two step approach by, first, extracting the spatially resolved signals, and, second, applying a user identification. In the first step, the uplink (pre-)beamformer, we use a conventional beamforming algorithm. For the second step, the classification of the spatially resolved signals, we need a user identifier. In case of GSM the midamble serves this purpose. We note in passing that GSM is not an ideal test case in this respect, because the cross-correlation of different midamble sequences is low.

Failures of the DOA estimation and false user identifications can be fatal. We will introduce a tracking concept that remedies erroneous decisions of the processing steps executed before the tracker: This will relax the requirements on the algorithmic front end, i.e., the DOA estimation and DOA identification. To be compatible with frequency hopping, only the user DOAs may be tracked. We will demonstrate how the tracking increases the robustness against interference.

Now, reliable user directions are available allowing final beamforming. The question remains whether dedicated interferer nulling is essential or not. If so, the nulling strategy will have to be compatible with the mobile radio channel and the limited number of degrees of freedom (number of antenna elements). We will consider "broad nulls" for this purpose.

We will address critical questions concerning the required angular resolution of the DOA estimator, how to separate a wanted signal from an interfering one, and whether dedicated interferer nulling is required or not.

\section{The AdAPtive Antenna Array Processor A ${ }^{3} \mathrm{P}$}

The array processing (Fig. 3) is based on a DOA estimation (DOAE). For each estimated DOA we extract, with the uplink beamformer (ULBF), a spatially resolved signal (uplink spatial pre-filtering), containing only the GSM midamble as a training sequence ( 26 bits). These spatially resolved midambles are then fed to the user identification (UID) that decides whether a DOA

${ }^{1}$ Following this argumentation could lead to the conclusion that a switched beam system with a user identification would already suffice. This is not the case. The advantage of a switched beam system is that only a single transceiver train is required [19]. But then only a very limited choice of beams is available for the final beamforming. Especially null steering is not possible with a switched beam system. 


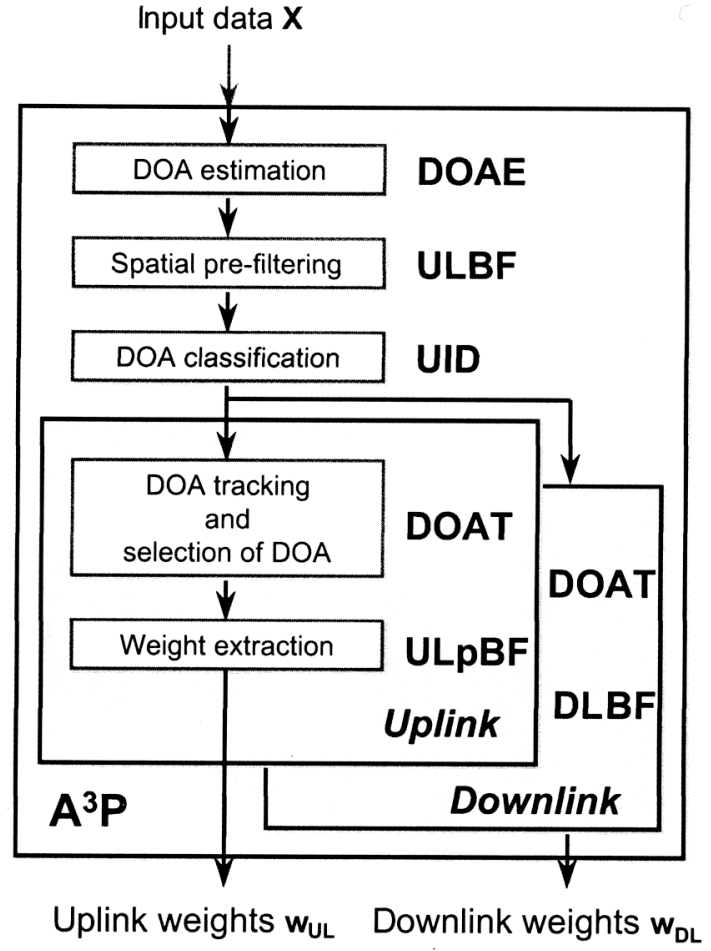

Fig. 3. Adaptive Antenna Array Processor $A^{3} P$. DOAE ... DOA estimation, ULBF ... uplink beamformer, UID ... user identification, DOAT ... DOA tracking, ULpBF . . . uplink post beamformer, DLBF . . downlink beamformer.

belongs to a user or to an interferer. The so identified user DOAs are the input to an uplink and to a downlink tracker (DOAT). The tracked user DOAs and the interferer DOAs are used to determine the weight vectors for the final post beamforming. This beamforming algorithm puts a main beam into the wanted user direction, while placing broad nulls into the direction of the interferers and maintaining a low sidelobe level. In the following we will discuss each of the subprocedures (DOAE, ULBF, ...) in more detail.

\section{A. DOA Estimation}

Estimating the DOAs is a well-known problem in signal processing [11]. The input to the estimator is the calibrated baseband matrix of measured data

$$
\mathbf{X}=\left[\begin{array}{llll}
\mathbf{x}_{1} & \mathbf{x}_{2} & \cdots & \mathbf{x}_{N}
\end{array}\right],
$$

where $\mathbf{x}_{n}, 1 \leq n \leq N=148$, is a column vector with $M$ elements corresponding to the $n$-th temporal snapshot of the antenna array. $M$ is the number of sensors. A baseband measurement matrix $\mathbf{X}$ corresponds to one GSM timeslot. ${ }^{2}$

We implemented three different algorithms, two subspace-based approaches and one spectral-based approach. The subspace-based algorithms are Unitary ESPRIT [7], and Unitary ESPRIT with subspace tracking.

Unitary ESPRIT estimates the signal subspace by means of an eigenvalue decomposition. From this estimated signal subspace the DOAs are calculated by solving the Invariance Equation and a subsequent spatial frequency estimation (see Table I). Instead

${ }^{2}$ In general, a lower-case boldface letter designates a column vector and a capital boldface letter a matrix.
TABLE I

SUMMARY OF THE SUBSPACE-BASED DOA EstimatoRs (EVD ... EIGENVALUE DECOMPOSITION

\begin{tabular}{c|c}
\hline Unitary ESPRIT & Unitary ESPRIT with subspace tracking \\
\hline \hline EVD & PASTd \\
\hline \multicolumn{2}{c}{ Solution of the Invariance Equation } \\
\hline \multicolumn{2}{r}{ Spatial frequency estimation } \\
\hline
\end{tabular}

of estimating the signal subspace, the subspace tracker PASTd (Projection Approximation Subspace Tracking and Deflation) [25] recursively tracks the signal subspace. In quasistationary channels the base of the signal subspace is only slowly timevarying. It is therefore more efficient to track those changes than to perform a full subspace estimation every burst. In both algorithms the model order, or the number of DOAs, is estimated by an information theoretic criterion such as Rissanen's MDL [22].

The third algorithm is a beamforming technique. Capon's Beamformer [3], also known as Minimum Variance Method (MVM), minimizes the power contribution from noise and any signals coming from other directions than $\phi$, while maintaining a fixed gain into the direction $\phi$.

The resulting spatial power spectrum is given by

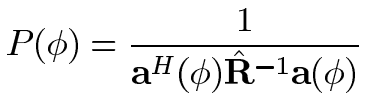

where $\mathbf{a}(\phi)=\left[1 e^{-j k d \cos \phi} \ldots e^{-(M-1) j k d \cos \phi}\right]^{T}$ is the uniform linear array steering vector, $k$ is the wavenumber, and $d$ the antenna element spacing. $\hat{\mathbf{R}}$ is the sample covariance matrix

$$
\hat{\mathbf{R}}=\frac{1}{N} \mathbf{X X}^{H} \text {. }
$$

A one-dimensional search in the spatial power spectrum $P(\phi)$ is necessary to find the DOAs.

After the DOAs, $\phi_{l}, 1 \leq l \leq L$, have been estimated we have to separate the user DOAs from the interferer DOAs. The $A^{3} \mathrm{P}$ considers all relevant paths that correspond to the user. Our system thus tries to identify all DOAs for the user and exploits this information to derive weight vectors for the final beamforming. Earlier mobile radio experiments have shown that a DOA estimator may fail if only a single DOA was considered for the user. In a typical cellular mobile radio channel this is not sufficient. In the actual implementation, we will utilize only one of the user directions, i.e., the strongest one. But having at hand more than a single user direction will lead to quickly switching to whichever is best.

The next two steps are required to categorize the DOAs found.

\section{B. Spatial Prefiltering}

The uplink beamformer ULBF extracts from $\mathbf{X}$ a spatially resolved signal for each of the $L$ estimated DOAs. Thus we have to derive $L$ weight vectors, $\mathrm{w}_{\mathrm{ULBF}, l}, 1 \leq l \leq L$, whose patterns steer a beam into the wanted directions $\phi_{l}$, while nulling all other directions. As weight matrix

$$
\mathbf{W}_{\mathrm{ULBF}}=\left[\begin{array}{llll}
\mathbf{w}_{\mathrm{ULBF}, 1} & \mathbf{w}_{\mathrm{ULBF}, 2} & \cdots & \mathbf{w}_{\mathrm{ULBF}, L}
\end{array}\right],
$$

we apply the Moore-Penrose pseudoinverse [10], designated by 
$\dagger$, of the estimated steering matrix.

$$
\mathbf{W}_{\mathrm{ULBF}}^{H}=\hat{\mathbf{A}}^{\dagger}
$$

where

$$
\hat{\mathbf{A}}=\left[\begin{array}{llll}
\mathbf{a}\left(\phi_{1}\right) & \mathbf{a}\left(\phi_{2}\right) & \cdots & \mathbf{a}\left(\phi_{L}\right)
\end{array}\right] .
$$

Thus each weight vector $\mathbf{w}_{\mathrm{ULBF}, l}$ is constructed to get a main beam into $\phi_{l}$ and nulls into all other estimated DOAs.

In a second step the spatially resolved signal vectors, $\hat{\mathbf{s}}_{l}, 1 \leq$ $l \leq L$, result from the uplink beamforming process

$$
\hat{\mathbf{s}}_{l}=\mathbf{w}_{\mathrm{ULBF}, l}^{H} \mathbf{X}_{\text {midamble }} .
$$

$\mathbf{X}_{\text {midamble }}$ is the part of the baseband measurement matrix $\mathbf{X}$ that contains the midamble (training sequence).

In the next step the spatially resolved midambles are fed to the user identification.

\section{User Identification}

The user identification UID is based on the detection of the spatially resolved midamble sequence, $\hat{\mathbf{s}}_{l}$, at bit level. By comparing the received midambles with the known user midamble, we calculate the number of bit errors within the training sequence. A spatially resolved signal, and thus the corresponding DOA, is attributed to a user, when the number of bit errors is smaller than a threshold. We so identify not only a single user path but all paths that correspond to the intended user, provided the signal quality is sufficient.

\section{DOA Tracker}

After the user identification, basically all information is at hand to direct the main beam into a user direction and to null the interferer DOAs: estimated and classified DOAs and the corresponding power values. Without the tracker, $A^{3} P$ exploits information from the current burst only, information from previous bursts is not yet used. We only track user DOAs, because interferer DOAs may change from burst-to-burst with frequency hopping, dynamic channel assignment, and discontinuous transmission (DTX).

The DOAT performs several tasks: averaging of estimated DOAs, assessing the reliability of an incident path, and selection of DOAs for final beamforming. A DOA is only selected if a minimum signal quality of an incident path persists over a certain period of time. By exploiting the reliability we improve the system's robustness. Each of these tasks is done separately for uplink and downlink, because the averaging in downlink requires larger memory length. A tracker is initiated for every incident path, containing its average DOA and its reliabililty. From the pool of trackers, each assigned to a multipath component, we select a single one to determine the main beam direction. Evidently, this can be done on a burst-to-burst basis, giving the possibility to instantaneously react to the current channel situation and thus to optimize the SNR gain.

Studying algorithms for DOA-based smart antennas, we found that the far-off estimates were the most detrimental. We found that the statistics of the estimated DOAs determined from measured data could not be rendered by a standard distribution

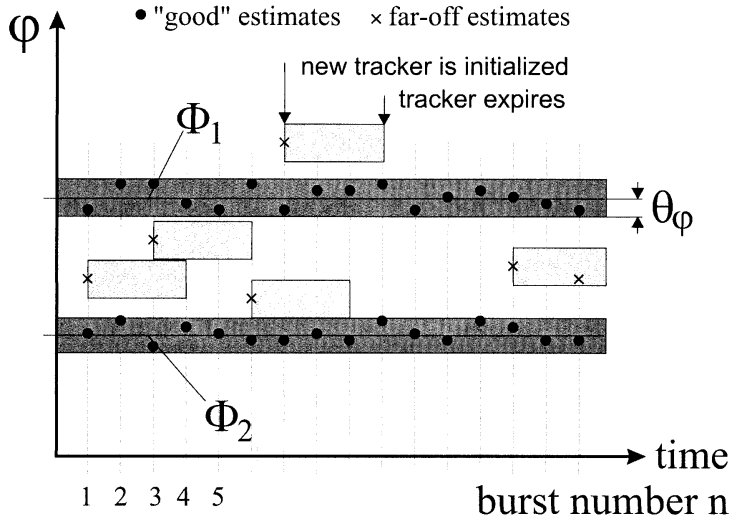

Fig. 4. User DOAs and trackers in an environment with two multipaths. In each burst the current user DOAs are applied to update the existing trackers. A tracker is updated only if a close-by user DOA is present in the current burst. If not, a new tracker is initialized.

function. Therefore, we concentrated on how to eliminate the far-off estimates. If the statistics cannot be modeled correctly, choosing independent trackers eliminates the wrong estimates.

Our tracking algorithm uses a bank of independent Kalman filters, based on a linear model in which the DOAs can change only with small angular velocity [4]. Each resulting tracker is equivalent to exponentially weighted averaging. The detailed choice of initialization parameters of the tracker was rather uncritical and had little influence on overall $C / I$ gain. The DOA estimate, $\Phi_{l}(n)$, provided by the tracking algorithm (in short "a tracker"), is updated with an estimate of a user DOA, $\hat{\phi}_{U, l}$, if $\left|\hat{\phi}_{U, l}-\Phi_{l}(n)\right|$ is below a threshold, $\theta_{\phi}$, which is typically some degrees. Thus we map each estimated DOA on a single tracker if it is close-by; we chose $\theta_{\phi}=5^{\circ}$. If there is no close-by tracker available, a new tracker will be initialized. Fig. 4 illustrates this effect. If such a tracker is not updated on a regular basis we assume it to be an artifact, caused either by an erroneous identification of a supposed user DOA or by far-off estimates. After 50 bursts (230 $\mathrm{ms}$ ) of not being updated the tracker expires and is deleted. Thus trackers of far-off estimates will vanish after some time (light shaded regions). This principle allows to suppress the influence of the far-off estimates on the final beamforming process. The trackers are independent of each other, guaranteeing that an artifact will not influence the other trackers at all.

The selection of the DOAs requires additional data that is collected for each tracker.

- Reliability:

Heuristic definition:

The reliability of a single tracker, $\Phi_{l}(n)$, is the total number of its updates.

We use this reliability to optimize robustness in the selection process.

- Instantaneous uplink power:

Although tracking implies averaging of the DOAs, we still allow instantaneous changes of the main beam direction by selecting the strongest DOA according to the instantaneous signal power of the incident waves. This introduces a kind of angular selection diversity into $A^{3} P$, but a tracked DOA is only selected if its reliability is large enough. The estimated user DOA $\phi_{U, l}$ corresponds to a spatially resolved signal $\hat{\mathbf{s}}_{l}$. The power of the spatially re- 
solved signal

$$
P_{l}=\hat{\mathbf{s}}_{l} \hat{\mathbf{s}}_{l}^{H}
$$

is the estimate for the instantaneous uplink power of the signal incident from $\phi_{U, l}$. Since $\phi_{U, l}$ updates the tracker $\Phi_{l}, P_{l}$ is attributed to the $l$ th tracker.

- Average uplink power:

For the downlink we base the selection of user DOAs on average power values measured in uplink, i.e., we assume that the average power value (and therefore the mean path loss) is the same in uplink and downlink.

\section{E. Signal Reconstruction-Final Beamforming}

We apply beamforming algorithms in uplink and in downlink that place a main beam to the selected user DOA and broad nulls [20] to the $\tilde{L}-1$ directions of the interferers. $\tilde{L} \leq L$ is the number of selected directions, consisting of a single direction for the main beam and $\tilde{L}-1$ directions of the interferers. Note that the situation differs significantly from the pre-spatial filtering (ULBF). Now, after UID, we know whether a DOA belongs to a user or to an interferer. Also, the tracker has rendered the estimated DOAs more reliable.

1) Uplink Post Beamformer: For the uplink post beamformer ULpBF the DOAT has selected the user tracker (tracked DOA) with the strongest instantaneous power. By adaptation to the current fading situation in the uplink it thus implements angular selection diversity, exploiting any decorrelation of the two strongest paths belonging to the wanted user.

$A^{3} P$ could, in principle, extract separately the secondstrongest signal as well. As the base station has a diversity receiver, other forms of angular diversity combining are possible [23]. $A^{3} P$ was designed to be compliant with the existing diversity base station receiver without any further changes. We therefore delegated the combination of the signals from multiple beams to this diversity receiver. ${ }^{3}$ The maximum improvement to be gained by steering a second beam would have been the difference between selection combining (in our case angular selection diversity) and maximum ratio combining which amounts to $1.3 \mathrm{~dB}$ in SNR [9].

2) Downlink Beamformer: Downlink fading is, of course, unknown at the base station. Thus we can only use averaged information derived from the uplink. For transmission the DLBF forms a beam into the direction with the largest average power.

\section{Definition of $C / I$ GAIN AND SNR GAIN}

The basic benefits of a smart antenna are increased signal power and reduced interference. This will result in a reduced bit error rate, hence an improved service quality. To fully exploit $A^{3} P$ 's possibilities it is essential to understand how $A^{3} P$ increases the system performance. Only this will allow to assess in which environments $A^{3} P$ can be introduced with the largest possible gain. The bit error rate (raw or encoded) is a possible

${ }^{3}$ Because of the specific structure of dual soft-decision reception, a complex output signal after diversity reception was not available. Hence an output SNR or $\mathrm{C} / \mathrm{I}$ could not be calculated, which are the very quantities by which the smart antenna improvement could and should be assessed. This is the reason why we only deal with a single DOA in the assessment. measure to assess a system, but it depends on the applied base band detector, the type of service (voice or data), etc. Thus we will quantify $\mathrm{A}^{3} \mathrm{P}$ 's benefits based on the C/I gain and SNR gain. This has an additional advantage: we will be able to better understand how the interference suppression depends on the SNR gain and vice versa, which in turn allows deeper insight into the mechanisms of a smart antenna system.

We processed each measurement scenario to obtain the following values:

- The instantaneous input $\mathrm{SNR}, \mathrm{SNR}_{\mathrm{in}, m}(n), m=1 \ldots M$, $n=1 \ldots N_{\text {burst }}$, i.e., the input SNR at each antenna element $m$ for every burst $n$.

- The instantaneous output $\mathrm{SNR}, \mathrm{SNR}_{\text {out }}(n), n=$ $1 \ldots N_{\text {burst }}$, i.e., the output SNR after beamforming for every burst $n$.

- The instantaneous input $\mathrm{C} / \mathrm{I}, \mathrm{C} / \mathrm{I}_{\text {in }, m}(n), m=1 \ldots M$, $n=1 \ldots N_{\text {burst }}$, i.e., the input $\mathrm{C} / \mathrm{I}$ at each antenna element $m$ for every burst $n$.

- The instantaneous output $\mathrm{C} / \mathrm{I}, \mathrm{C} / \mathrm{I}_{\text {out }}(n), n=1 \ldots N_{\text {burst }}$, i.e., the output $\mathrm{C} / \mathrm{I}$ after beamforming for every burst $n$.

For every time slot we calculate an instantaneous SNR gain

$$
\Delta \operatorname{SNR}(n)=\operatorname{SNR}_{\text {out }}(n)-\frac{1}{M} \sum_{m=1}^{M} \operatorname{SNR}_{\text {in }, m}(n)
$$

i.e., we subtract from the output SNR the input SNR, averaged over the $M=8$ antenna elements. We define the beamforming gain $^{4}$ as the average over a sufficiently large number of the measured instantaneous SNR gain values $\left(N_{\text {sample }}=5000\right)$

$$
G_{b}=\frac{1}{N_{\text {sample }}} \sum_{n=1}^{N_{\text {sample }}} \Delta \operatorname{SNR}(n)
$$

A simple estimate of the beamforming gain is possible, because the beamformer tries to add the antenna inputs of the wanted signal in phase, which leads to an increase of the signal power by $M^{2}$ due to beamforming. At the same time, the noise floor is increased because each antenna has its own receiver. Assuming that these noise signals are uncorrelated, an $M$-fold increase of the noise power at the beamformer output is obtained. Therefore, the beamformer can improve the SNR by a factor of $\left(M^{2} / M\right)=M$ under ideal conditions. This corresponds to $10 \log M \mathrm{~dB}$ gain, e.g., a gain of $9 \mathrm{~dB}$ with eight antenna elements in the array. ${ }^{5}$

In line-of-sight scenarios [LOS, cf. Fig. 5(a)] the beamforming gain can actually be verified. In Fig. 5(a) we plotted the cumulative distribution functions (cdfs) of the eight input SNRs of an eight-element array using 5000 samples each. Additionally, the cdfs of the average input SNR and the output SNR after maximum ratio combining are given. A well-known result from the literature is that maximum ratio combining results in an output given as the sum of the input SNR values of

\footnotetext{
${ }^{4}$ This gain is sometimes also called array gain.

${ }^{5}$ In some beamformers, however, not only phase corrections are applied. In case of tapering the beamformer weights the antenna inputs not only in phase but also in amplitude to achieve controlled sidelobe levels of the beam pattern. The cost of tapering is a broader main beam and a reduction of the SNR improvement, since the increase of the signal power is no longer $M^{2}$ because some of the antenna inputs are attenuated. A similar degradation is obtained by inserting nulls into the antenna pattern which also requires some weight amplitudes to be modified.
} 


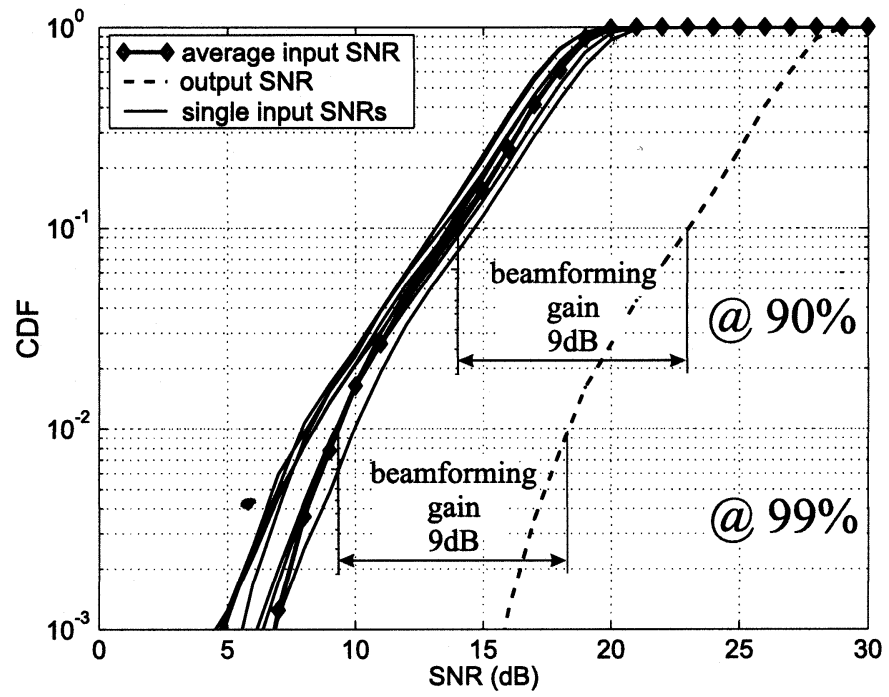

(a)

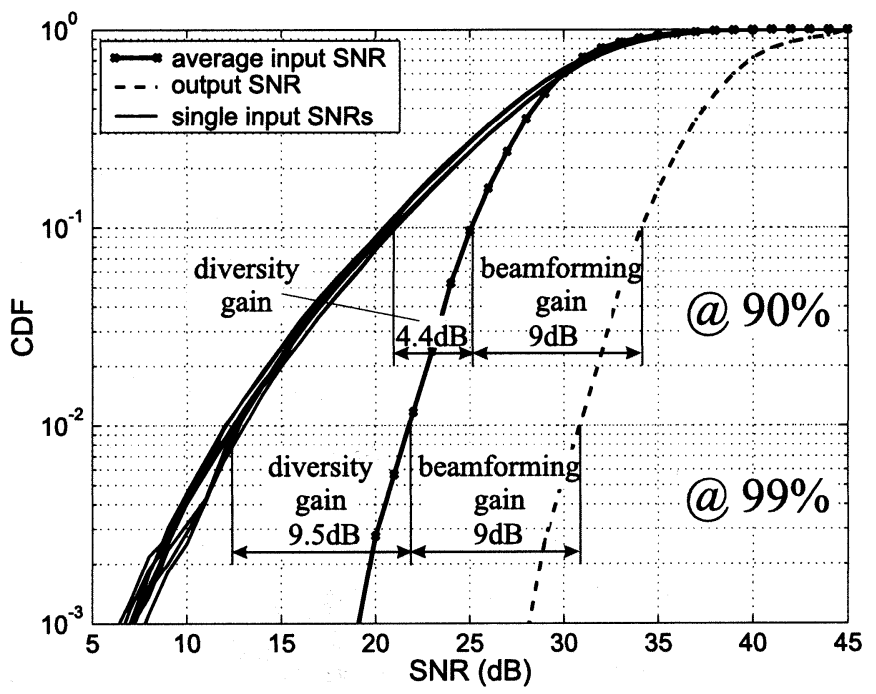

(b)

Fig. 5. Definition of the SNR gain. Cumulative distribution functions of the input, averaged input and output SNRs of measured exemplary statistics are presented. The total SNR gain is the sum of the beamforming gain and the diversity gain. (a) Line-of-Sight (NLOS) scenario. Here no diversity gain is available. (b) Non-line-of-sight (LOS) scenario.

the $M$ antenna elements. Since the signal envelopes received by the antenna elements are correlated and no diversity gain can be obtained, the SNR gain is equal to the beamforming gain of $9 \mathrm{~dB}$. Note that the input SNR and output SNR have the same slope - an indicator that no diversity gain is achieved.

Under non-LOS conditions the signal typically encounters multipath propagation with a certain angular spread of the incoming wave fronts. It can be shown, that the correlation between the input signal evelopes depends on the spread of the associated angles of arrival. An angular spread of $0^{\circ}$ corresponds to a single arriving wave front yielding perfect correlation between the envelopes and therefore only beamforming gain, but no diversity gain.

With an increasing angular spread the signal envelopes show increasingly decorrelated fading behavior. This decorrelation has two effects: A potential loss of beamforming gain and an in- crease of diversity gain. We can explain this as follows: Since the phases of the incoming signals are partly decorrelated, a chosen weight vector only yields perfect combining for a small period of time during which the channels are coherent. Since the signal phases change individually, the weight vector becomes less appropriate over time and needs regular updates. Therefore, there is a potential loss in beamforming performance which can be compensated by more frequent adaptations of the weight vector. The second effect is the decorrelation of the signal amplitudes which are typical for diversity scenarios.

Both effects can be observed in typical NLOS scenarios. An example is given in Fig. 5(b), where the average input SNR curve already displays a gain. Due to the decorrelation the input signals fade individually. Hence, the situation that all the input SNR values are small at the same time is quite rare, and therefore the probability of small average input SNRs is low. This yields an increasing gain for decreasing values of the cdf curve, which we define to be the diversity component of the SNR gain. On top of that, a beamforming gain close to the theoretical optimum of $9 \mathrm{~dB}$ with eight antenna elements is obtained independently of the probability level. Note that the results show that a diversity gain is achieved since the slopes of the input and output SNR cdf are now different.

Since these effects should be covered by an appropriate definition for the SNR gain (and also the $C / I$ gain), this leads us to the following definitions:

Definition: The SNR gain and the $C / I$ gain at the $m$ th antenna element at a certain probability level, $\gamma$, are

$$
\begin{aligned}
& \Delta \mathrm{SNR}_{m}^{\gamma}=\left.\mathrm{SNR}\right|_{\mathcal{C}_{\text {out }} \mathrm{SNR}_{=1-\gamma}}-\left.\mathrm{SNR}\right|_{\mathcal{C}_{\text {in }, m}} \mathrm{SNR}_{=1-\gamma}, \\
& \Delta C / I_{m}^{\gamma}=\left.\mathrm{SNR}\right|_{\mathcal{C}_{\text {out }}^{C / I}=1-\gamma}-\left.\mathrm{SNR}\right|_{\mathcal{C}_{\text {in }, m}^{C / I}=1-\gamma} .
\end{aligned}
$$

We define the SNR gain, $\Delta \mathrm{SNR}^{\gamma}$, and $C / I$ gain, $\Delta \mathrm{C} / \mathrm{I}^{\gamma}$, as the average over all antenna elements

$$
\begin{aligned}
\Delta \mathrm{SNR}^{\gamma} & =\frac{1}{M} \sum_{m=1}^{M} \Delta \mathrm{SNR}_{m}^{\gamma} \\
\Delta \mathrm{C} / \mathrm{I}^{\gamma} & =\frac{1}{M} \sum_{m=1}^{M} \Delta \mathrm{C} / \mathbf{I}^{\gamma} .
\end{aligned}
$$

$\mathcal{C}_{\text {in }, m}^{\mathrm{SNR}}, \mathcal{C}_{\text {out }}^{\mathrm{SNR}}, \mathcal{C}_{\text {in }, m}^{\mathrm{C} / \mathrm{I}}$, and $\mathcal{C}_{\text {out }}^{\mathrm{C} / \mathrm{I}}$ are the cdf of the input and output SNR and $C / I$ values.

In this work we choose two values for the probability level: $\gamma=$ $99 \%$ and $\gamma=90 \%$. A result is then interpreted as: The SNR gain, $\Delta \mathrm{SNR}^{99 \%}$ at the $1 \%$ cdf probability level(corresponding to an probability level of $\gamma=99 \%$ ), is the difference of the input and output SNR at the corresponding probability level (see Fig. 5).

Having defined the quality measures for a smart antenna system, we will evaluate $\mathrm{A}^{3} \mathrm{P}$ in detail.

\section{MEASUREment Modeling-Generating ScEnarios}

For the assessment of the complete system in uplink we used actual transmitted data. We recorded the received data of a large number of measurement runs with $M=8$ antennas, covering a period of about $20 \mathrm{~s}$ each. This corresponds to $N_{\text {burst }}=5000$. To model interference with a single mobile station in the field, we superimposed several (typically two) of these measurement 


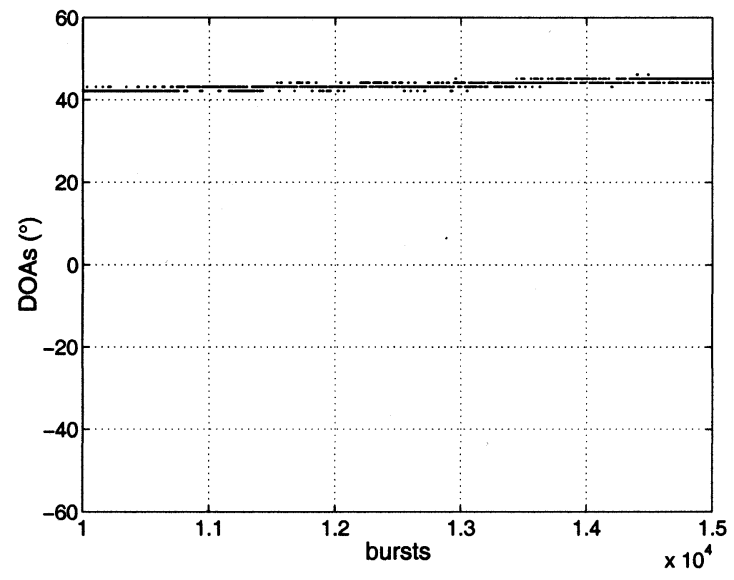

(a)

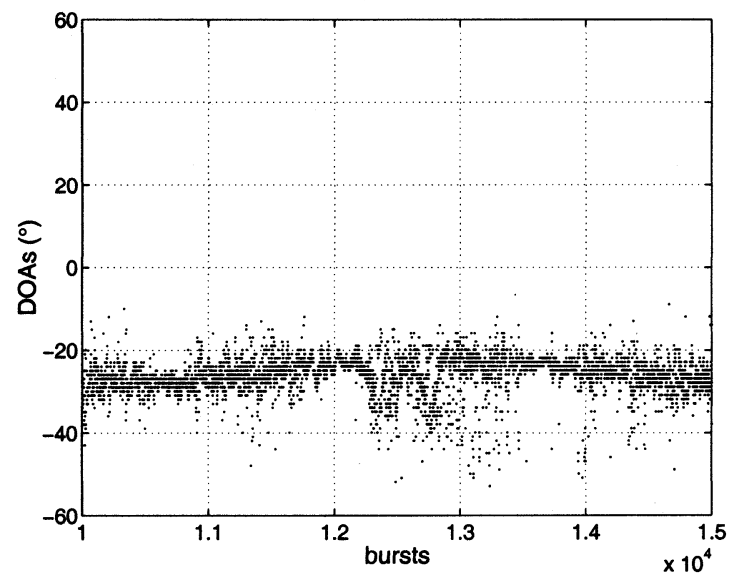

(b)

Fig. 6. DOAs, resulting from the scanning beam algorithm of Scenario A. (a) The user signal had a small angular spread and (b) the interferer signal had a large angular spread.

runs and processed the data off-line. In fact, this is the only way to exactly diagnose $\mathrm{C} / \mathrm{I}$. This strategy leaves room for repeated processing of the same data set to compare different configurations of $A^{3} P$.

We set up two scenarios: Scenario A, favorable for a DOAbased smart antenna and a challenging Scenario B with overlapping DOAs. In both cases interfering signals with, on average, the same power as the user signal were present.

Scenario A consisted of a user signal with LOS to the BS (only slightly obstructed by trees) and an interferer signal with quasi-LOS to the BS (see Fig. 6). Both signals were well separated in angle. While the user signal had a small angular spread, we observed a significant spread for the interferer (the MS was moving close to buildings, leading to local scattering). The input $\mathrm{C} / \mathrm{I}$ was less than $0 \mathrm{~dB}(-5 \mathrm{~dB})$ in about $50 \%(10 \%)$ of the cases.

To challenge $A^{3} P$ we selected Scenario $B$ in which the user and interferer signal have large angular spreads and arrive partly from the same angular ranges (see Fig. 7). The user signal had two multipaths around $\phi_{1}=20^{\circ}$ and $\phi_{2}=45^{\circ}$. The interferer signal had an overlap around $\phi_{2}$ in most bursts. The input C/I was less than $0 \mathrm{~dB}(-13 \mathrm{~dB})$ in about 50\% (10\%) of the cases.

The DOAs plotted in Figs. 6 and 7 were extracted from the signals by applying a simple Fourier-based scanning beam al- gorithm and taking, at each burst, the DOA that gives largest output power.

\section{Angular Resolution of DOA Estimator}

First, we want to find the most appropriate DOA estimator, second, we study which angular resolution is required for smart antennas in public cellular mobile radio.

In the beginning, we use Scenario A (see Fig. 6) to quantify the maximum achievable $\mathrm{C} / \mathrm{I}$ gain, and to study the influence of the angular resolution of the DOA estimators. The $C / I$ gain was very high (Table II). The interference could be suppressed by as much as $22 \mathrm{~dB} .{ }^{6}$ The beamforming gain was up to $G_{b}=7 \mathrm{~dB}$. That $\mathrm{A}^{3} \mathrm{P}$ does not reach the optimum beamforming gain is mainly a consequence of the tapering, i.e., beamforming weights that have amplitude smaller than unity. ${ }^{7}$ As expected, there was practically no diversity gain available. This is a consequence of the small angular spread of the user signal.

Comparing the various DOAE estimators, we found that the $C / I$ gain in Scenario $\mathrm{A}$ is nearly independent of the algorithm's choice (Table II). Some larger differences in the SNR gain values are present, especially at the 99\%-level. If Unitary ESPRIT is applied, the $\Delta \mathrm{SNR}^{99 \%}$ is significantly degraded. Now a discussion of the actually relevant parameters for DOA-based smart antenna processing is in place.

Estimation accuracy is the first quantity we investigate. However, during the development of the array processing it turned out that not the accuracy is of main concern, but the robustness is. We thus will define a quantity that allows quantification of the estimation robustness and present the robustness of the implemented DOA estimators.

\section{A. DOA Estimation Accuracy}

To study accuracy, we first define the DOA estimate variation, $\sigma_{\mathrm{DOAE}}$, as the standard deviation of the estimated DOAs, $\hat{\phi}_{n}$, $n=1 \ldots N_{\text {samples}}$, when a single plane wave is incident; we used $N_{\text {samples }}=5000$.

We measured the estimate variation in a controlled LOS environment [13]. When a discrete wave was incident from broadside the measured estimate variation was smaller than $1^{\circ}$ for an input SNR larger than $0 \mathrm{~dB}$, but decreased monotonically to the order of some hundreds of a degree with increasing SNR up to $40 \mathrm{~dB}$, for all three estimators (see Fig. 8).

\section{B. DOA Estimation Robustness}

The estimate variation quantifies the accuracy of a DOA estimator, i.e., how accurate a DOA can be estimated under optimum conditions. However, the estimate variation has little meaning in mobile radio channels. Quantifying the robustness of a DOA estimator is a more challenging task. Different properties influence the performance of an estimator, like the angular spread, the number of clusters, the number of signal sources present, and the number of estimated DOAs. Thus

\footnotetext{
${ }^{6}$ If we exchanged the role of user and interferer signals we even obtained 25 $\mathrm{dB}$. Of course, $\Delta \mathrm{C} / \mathrm{I}^{\gamma}$ was higher when the angular spread of the interferer was smaller.

${ }^{7}$ To allow antenna weights to deviate from unity makes $A^{3} P$ capable of suppressing interference by low sidelobe level and broad nulls. The penalty paid is a reduced SNR gain.
} 


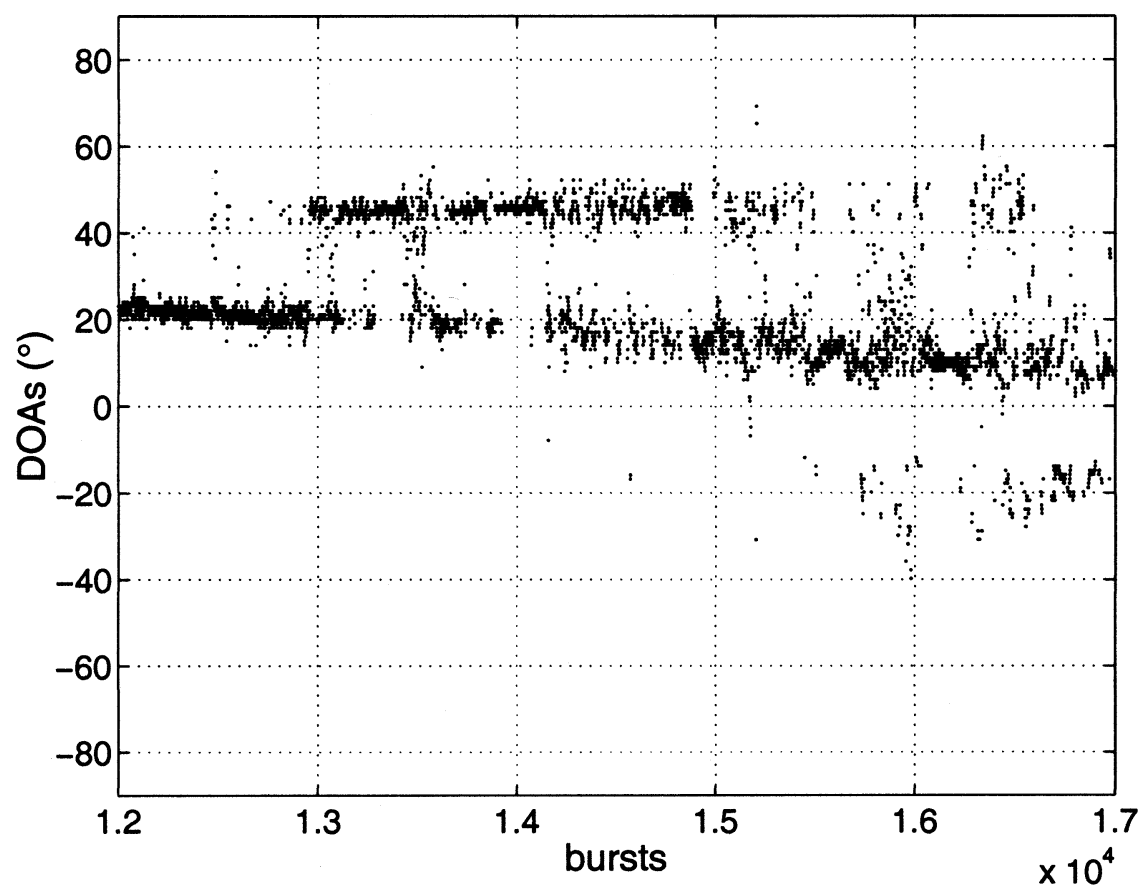

(a)

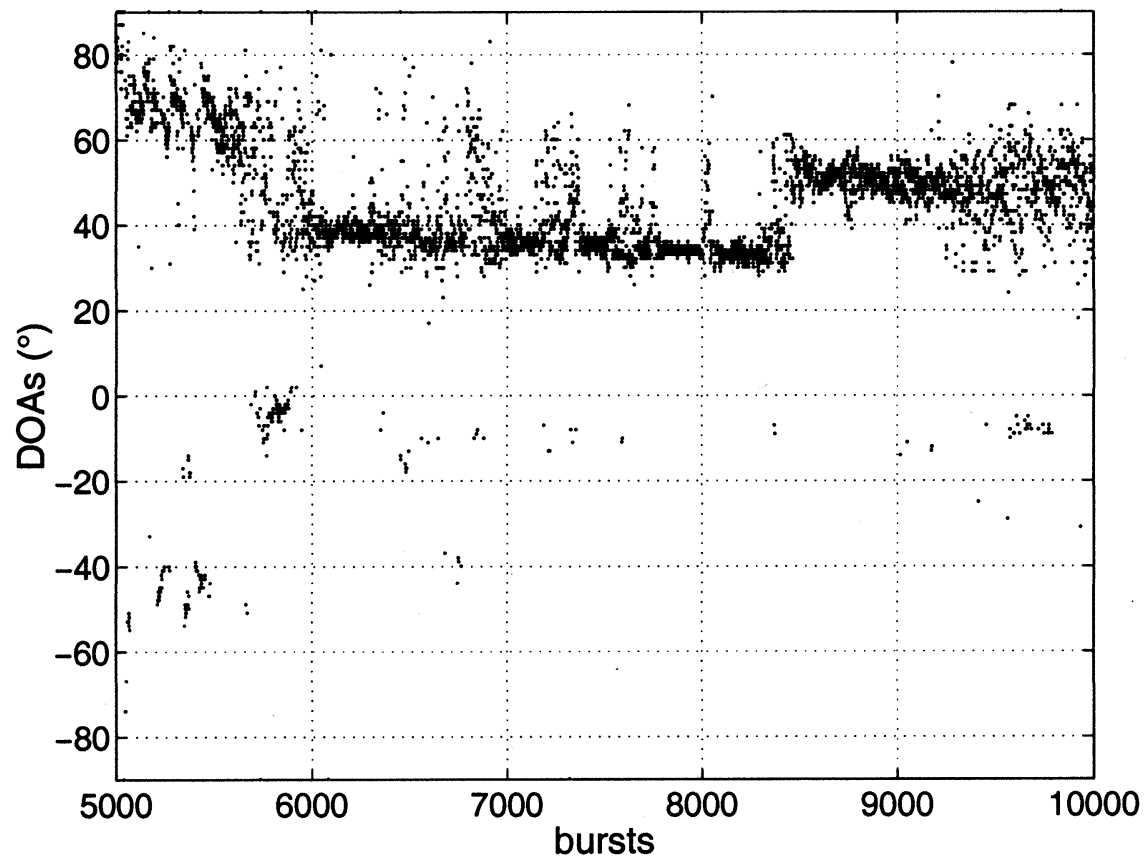

(b)

Fig. 7. DOAs, resulting from the scanning beam algorithm of Scenario B: In Scenario B, (a) the user and (b) interferer signals partly overlap in angle, thus leading to a challenging scenario.

an additional measure for the robustness of the estimator is needed: the estimation range.

To quantify the estimation range we determine the cdf, $\operatorname{cdf}_{\phi}$ of the estimated DOAs. When a single (nominal) DOA is incident, the estimation range is defined as the minimum size of the angular range around a nominal DOA, $\phi_{0}$, that includes $\alpha=38.3 \%$ of the estimated DOAs, $\phi$ (see Fig. 9).

$$
Q_{\mathrm{DOAE}}=\min _{\zeta}\left\{\phi\left(\operatorname{cdf}_{\phi}=\zeta\right)-\phi\left(\operatorname{cdf}_{\phi}=\zeta-\alpha\right)\right\}
$$

where

$$
\phi\left(\operatorname{cdf}_{\phi}=\zeta\right)<\phi_{0}<\phi\left(\operatorname{cdf}_{\phi}=\zeta-\alpha\right) .
$$

This condition assures that the nominal DOA stays within the defined angular range. It does not require symmetry around $\phi_{0}$.

- The definition allows a calculation from sample data, either measured or simulated.

- If a single (nominal) DOA is incident and the estimated DOAs are Gaussian distributed, $Q_{\mathrm{DOAE}}=\sigma_{\mathrm{DOAE}}$, i.e., 
TABLE II

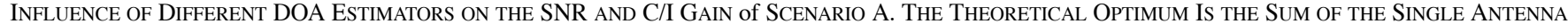
ELEMENT SNRS, CORRESPONDING TO MAXIMUM RATIO COMBINING WHEN NO INTERFERENCE IS PRESENT AND THE CHANNEL IS KNOWN

\begin{tabular}{c||c|c|c|c|c}
\hline \multicolumn{1}{c||}{ Configuration } & \multicolumn{5}{c}{ Scenario A } \\
\hline All values in dB & $\Delta C / I^{90 \%}$ & $\Delta C / I^{99 \%}$ & $\Delta S N R^{90 \%}$ & $\Delta S N R^{99 \%}$ & $G_{b}$ \\
\hline \hline Unitary ESPRIT & 22.1 & 21.2 & 6.8 & 3.9 & 6.7 \\
\hline PASTd & 22.1 & 22.1 & 7.0 & 6.7 & 6.8 \\
\hline PASTd, w/o burst-to-burst track. & 22.0 & 21.8 & 7.0 & 6.0 & 6.8 \\
\hline standard, MVM & 21.9 & 21.7 & 7.2 & 7.6 & 7.0 \\
\hline theoretical optimum & - & - & 9.3 & 9.8 & 9.0 \\
\hline
\end{tabular}

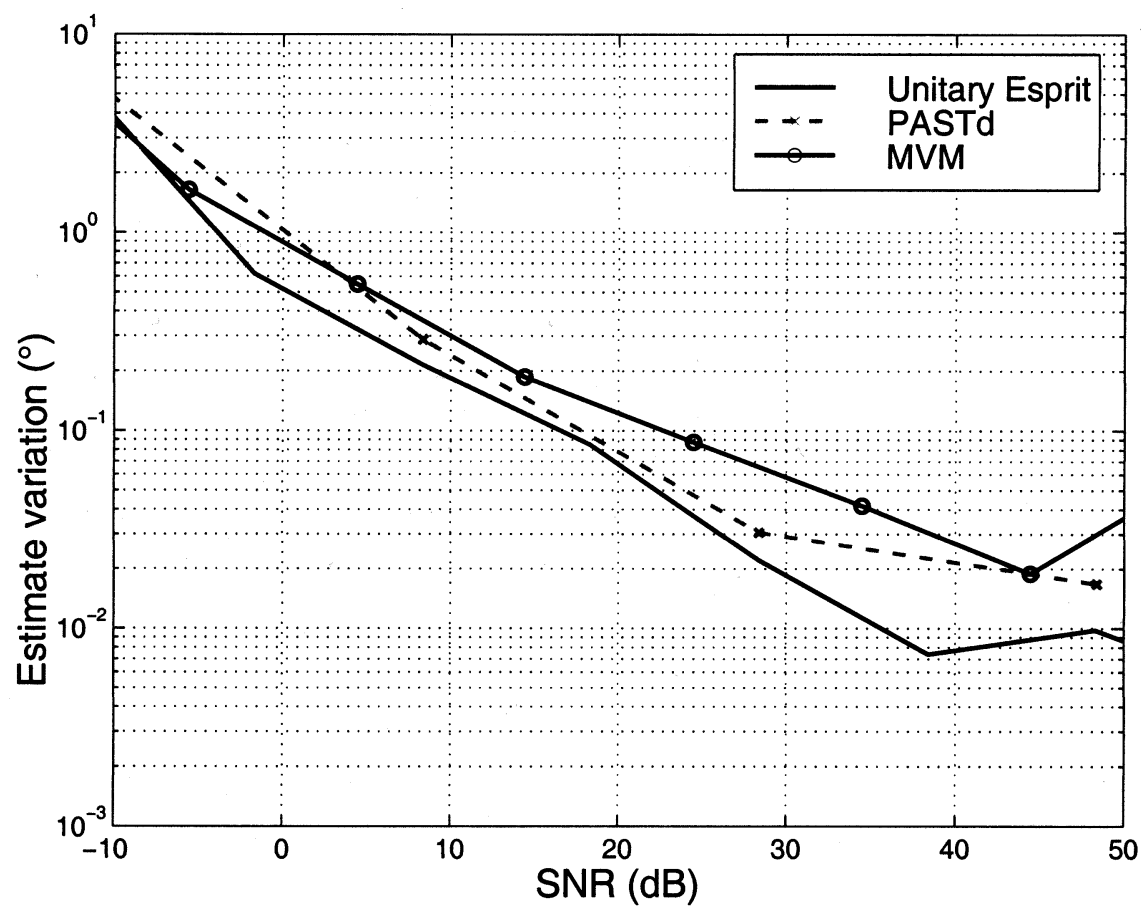

Fig. 8. Measured estimate variation of the DOAE versus SNR when a single plane wave is incident from $\phi=0^{\circ}$.
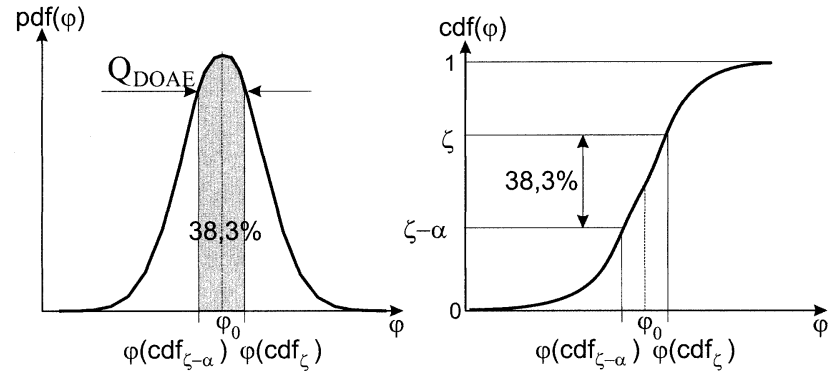

Fig. 9. Definition of the estimation range. A single nominal DOA $\left(\phi_{0}\right)$ with standard deviation $\sigma$ is present.

the estimation range is equal to the estimate variation, which explains why we set $\alpha=0.383$.

- Computing $Q_{\mathrm{DOAE}}$ requires, in addition to the sample estimates, only the knowledge of a nominal DOA, not the actual one.

- The concept can be easily generalized to the case of more incident DOAs [12].

To quantify the robustness of the implemented DOA estimators we applied them to a synthetic channel model, the GSCM
[15]. ${ }^{8}$ We assumed two active signal sources, each having local scatterers, Gaussian distributed around the mobile stations. This resulted in an rms angular spread of $2.5^{\circ}$ for each MS. Table III summarizes the channel parameters.

We varied the power of the transmitted signals, thus varying the input $C / I$. The number of estimated DOAs is fixed to $\hat{L}=2$, thus this assessment does not include the effect of nonideal rank estimation. Note that for MVM $\hat{L}$ cannot be fixed beforehand, because it is implicitly estimated.

Fig. 10 shows the surprising result of our simulation. The estimation range of MVM is the best, closely followed by PASTd with burst-to-burst tracking. Unitary ESPRIT and PASTd without burst-to-burst tracking perform equally, but worse than the other two.

The superior robustness of MVM is even clearer when we consider different angular spreads. Fig. 11 presents the estimation range for the same scenario, but the angular spread of each nominal DOA was varied. Especially at large angular spread, Unitary ESPRIT and PASTd often fail by producing far-off

${ }^{8}$ In contrast to all other assessments in this paper, we rely here on pure synthetic data, i.e., on a computer experiment. 


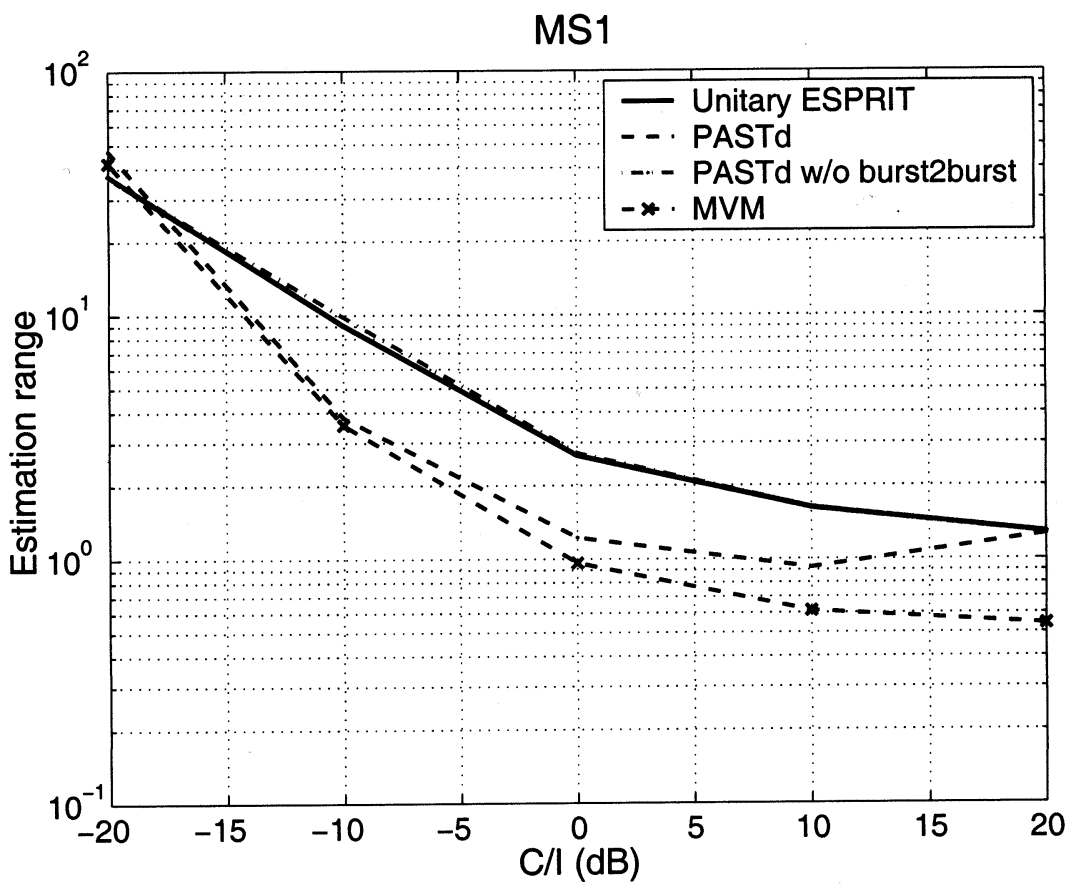

Fig. 10. DOA estimation range, $Q_{\mathrm{DOAE}}$, of Mobile 1 (see Table III) as a function of the average input $C / I$ for different DOA estimators.

TABLE III

Geometry-Based Stochastic ChanNel Model (GSCM) CHANNEL Parameters. Two MobiLes Are Present

\begin{tabular}{c||c|c}
\hline Parameter & Mobile 1 & Mobile 2 \\
\hline \hline nominal DOAs & $45^{\circ}$ & $-10^{\circ}$ \\
\hline number of local scatterers & 50 & 50 \\
\hline local scatterer radius & $100 \mathrm{~m}$ & $100 \mathrm{~m}$ \\
\hline distance MS-BS & $2000 \mathrm{~m}$ & $2000 \mathrm{~m}$ \\
\hline \hline rms angular spread of each multipath & $2.5^{\circ}$ & $2.5^{\circ}$ \\
\hline
\end{tabular}

estimates, and thus the estimation range is larger. In contrast, MVM can cope even with large angular spreads.

Based on the estimation range we conclude that MVM is the most robust estimator, when the signals are incident with a finite angular spread. The most robust estimator guarantees the best overall results also, not the most accurate one (Unitary ESPRIT). Evidently, the estimate range is a suitable quantity for the assessment of smart antenna systems.

To answer the question which DOA estimation resolution is necessary, we applied MVM with different angular resolution settings to Scenario A. ${ }^{9}$ The results are illustrated in Fig. 12. A performance degradation was only noticeable, if the angular resolution, $R_{f}$, was as large as $10^{\circ}$. This is no surprise, because $A^{3} P$ 's tracking algorithm was introduced to cope with a deficient estimation quality. Here, the tracker and the broad nulls in the beamforming are responsible for the excellent robustness: First, the tracker renders the DOA estimates more reliable, by

\footnotetext{
${ }^{9}$ To minimize complexity we perform the one-dimensional search in the spatial power spectrum in two steps: First, we find the peaks in a spectrum with angular resolution $R_{c}$. This first search in the "coarse" spectrum yields initial estimates for the DOAs. In a second, refined search we only look, with increased angular resolution, $R_{f} \leq R_{c}$, in the vicinity of the initial estimates. Thus, the final DOA estimates are available with fine resolution, $R_{f}$.
}

smoothing the estimated DOAs. Second, the broad nulls make sure that certain DOA errors will not cause the $C / I$ to drop, because interference is still suppressed sufficiently.

The SNR gain was reduced significantly in Scenario A when $R_{f}=10^{\circ}$. The narrow main beam and a small angular spread of the user signal in Scenario A caused a significant power (SNR) loss, when the user DOA was shifted by some degrees.

We conclude that $A^{3} P$ does not require DOA estimates with very high resolution. Instead it is more important that the estimators have high estimation robustness.

\section{ASSESSMENT OF TRACKING ALgORITHM}

If user and interferer signals overlap in angle the tracking algorithm plays a key role in increasing the robustness and thus improving $A^{3} P$ 's overall performance. We select Scenario B to demonstrate this improvement.

\section{A. Absolute SNR and C/I Gain}

For the "standard" configuration (MVM, tracking activated), the $\mathrm{C} / \mathrm{I}$ gain in Scenario B was still very high, $\Delta \mathrm{C} / \mathrm{I}^{99 \%}=18.5$ $\mathrm{dB}$ (see Table IV)! This is an excellent result, especially considering how challenging a situation this is for a DOA-based smart antenna. The SNR gain was low because all degrees of freedom were used up to suppress the significant interference, but we still achieve a beamforming gain of $4 \mathrm{~dB}$.

\section{B. Effect of Tracking}

The tracker is the key element in the $A^{3} P$ that guarantees system robustness. We will prove this by assessing Scenario B with and without a tracker (see Fig. 13). If the tracker was deactivated, the difference to the standard configuration had two effects: $A^{3} P$ could not cope with far-off estimates and most importantly, it could not remedy erroneous identifications of the UID. Finally, $A^{3} P$ aimed the main beam in the direction of the 


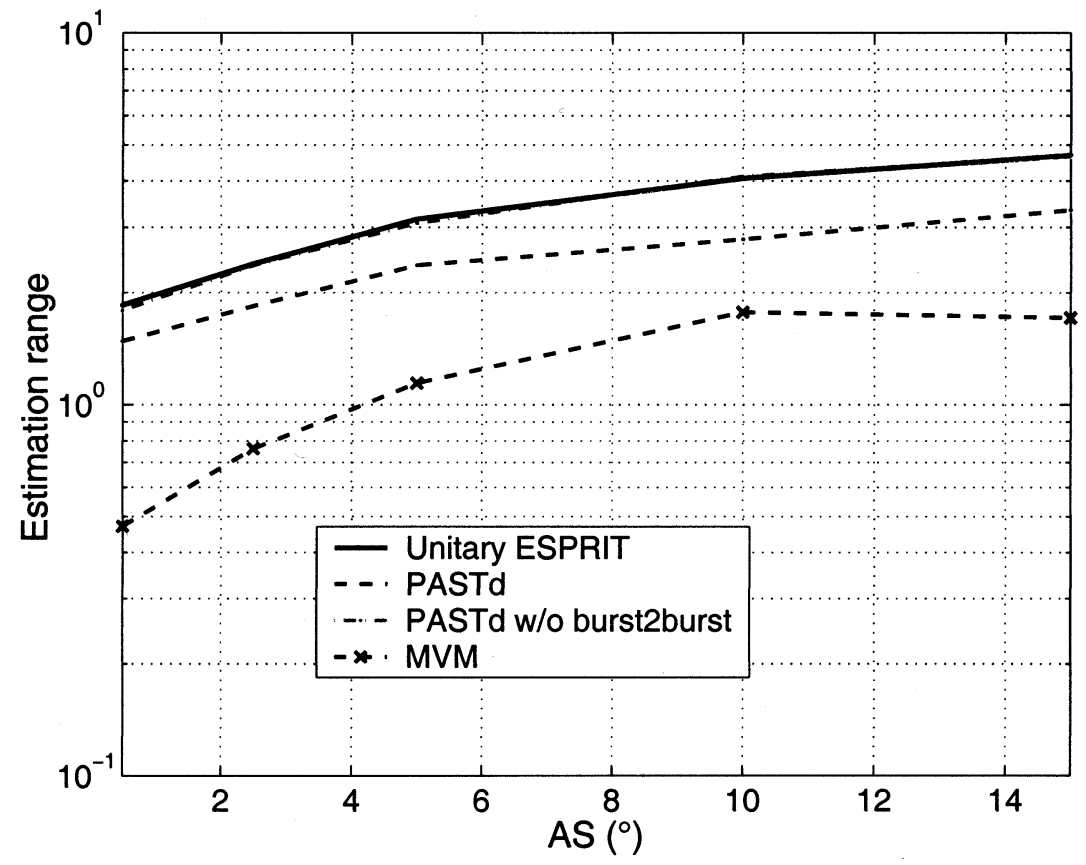

Fig. 11. DOA estimation range, $Q_{\mathrm{DOAE}}$, of Mobile 2 (see Table III) as a function of the angular spread, AS. The average input $C / I$ is $0 \mathrm{~dB}$.

strongest user DOA, but did not include any additional measures to increase the reliability of this decision. Deactivating the tracker reduced the $C / I$ gain by a tremendous $17.6 \mathrm{~dB}(5.6 \mathrm{~dB})$ at the $99 \%$ (90\%) probability level in Scenario B ${ }^{10}$ (see Fig. 13).

\section{Is Tracking Just Averaging?}

It is of interest to investigate whether averaging of the DOAs or increasing the robustness by selecting only DOAs with high enough reliability has larger influence on the performance. So we varied the averaging constant of the tracker, but calculated and used all other information, like the reliability. It turned out that even if we did not apply any DOA averaging at all, but still measured the reliability, the $\mathrm{C} / \mathrm{I}$ gain was only reduced by $0.5 \mathrm{~dB}$. Thus the reliability concept increases the robustness considerably. It is much more important for the $\mathrm{C} / \mathrm{I}$ gain than averaging the DOAs.

\section{BEAMFORMING STRATEGY}

The beamforming algorithms (ULpBF in uplink and DLBF in downlink) calculate the actual applied antenna patterns, i.e., the weight vectors. From previous simulations [14] we had concluded that it was of advantage to place broad nulls into the direction of the interferer DOAs to cope with a possibly large angular spread of the interference. To test this, we applied two different beamforming algorithms to measurement Scenario A: the beamformer SmearR [20] (broad nulling) and the Pseudo Inverse (conventional, sharp nulling).

The $\mathrm{C} / \mathrm{I}$ gain for the two beamforming strategies differs only by up to $0.7 \mathrm{~dB}$, the SNR gain is about $1.4 \mathrm{~dB}$ larger for the sharp nulling strategy (see Table V), a trend found also in other scenarios. This result demonstrates that there is nearly no improvement in the $\mathrm{C} / \mathrm{I}$ gain by broad nulling over the conventional nulling. From these results we would conclude that it is

\footnotetext{
${ }^{10}$ The effect was, of course, much less pronounced for Scenario A. There the $\mathrm{C} / \mathrm{I}$ gain differed only by about $0.3 \mathrm{~dB}$.
}

not worth placing broad nulls instead of sharp nulls, but broad nulling does make sense as we will see now.

\section{A. Broad Nulls and the DOA Estimation}

Intuition tells us that a broad null will reduce demands on the DOA estimation resolution. The idea is that if we can place a broad null into the direction of an interferer, the interferer DOA does not have to be known so exactly, as long as the angular spread of the interferer is smaller than the null width. With a broad nulling strategy we can also reduce the demands on the calibration procedure. Calibration errors cause shifted nulls, which, with respect to the interferer suppression, has a similar effect than inaccurate interferer DOAs. An improved robustness against calibration errors is especially in the downlink of importance, where calibration is more difficult than in uplink. ${ }^{11}$

Following our first argument (robustness against inaccurate interferer DOAs) we applied the MVM to Scenario A with different angular resolution settings and present the $\mathrm{C} / \mathrm{I}$ gain in Fig. 14. Increasing the angular resolution of the DOA estimation has a similar effect as an "artificial" shift of the nulls. The C/I gain decreases less for the broad nulling strategy! The C/I gain drops significantly for the Pseudo Inverse, when the fine resolution is larger than $R_{f}=1^{\circ}$, while for the beamformer with broad nulls the gain stays constant until $R_{f}=5^{\circ}$. Thus we conclude that broad null beamforming is robust against poorly estimated interferer DOAs.

\section{DisCUSSION OF CONCEPT AND CONCLUSION}

From the evaluation of the smart antenna we will now discuss the essential ingredients for a successful design of a DOA-based smart antenna. Note that such a processor is suitable for both upand downlink operation.

\footnotetext{
${ }^{11}$ In the uplink the smart antenna senses $M$ antenna signals that have passed the receiver and hence can be used for calibration purposes. In the downlink no such signals that have passed transmitter are available at first place.
} 


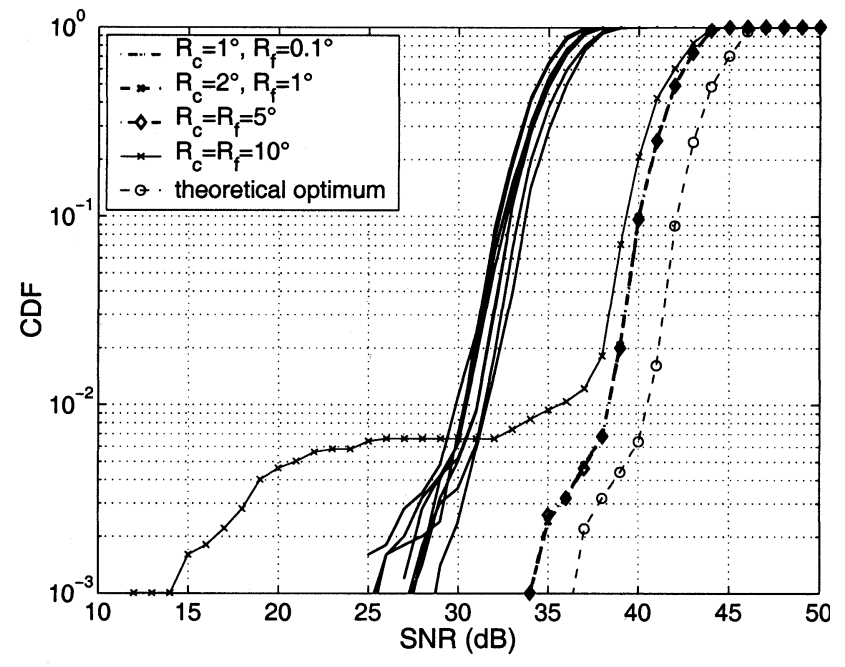

(a) Statistic of SNR.

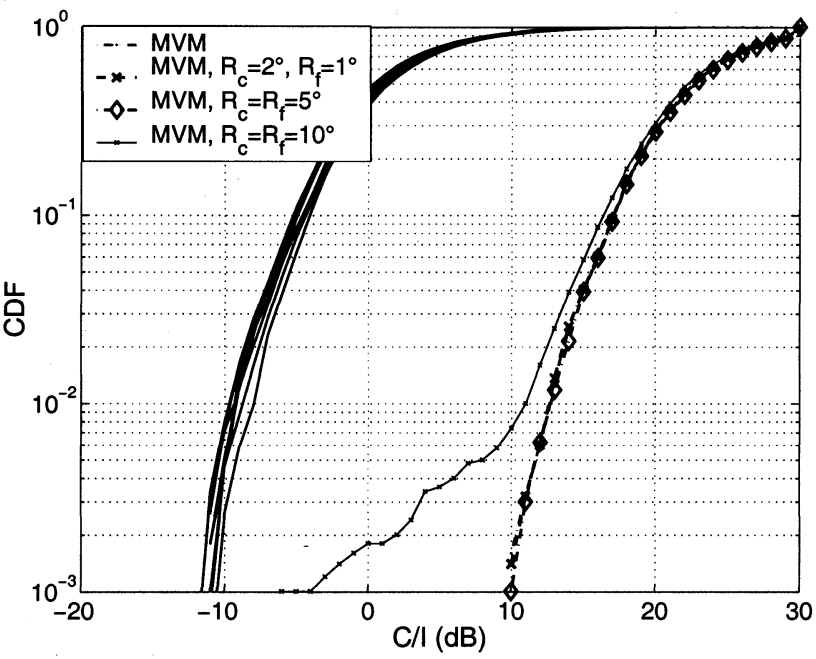

(b) Statistic of C/I.

Fig. 12. Influence of MVM's angular resolution, $R_{f}$, on the SNR (a) and $C / I$ (b) statistics in Scenario A. The theoretical optimum in (a) is the sum of the single antenna element SNRs, corresponding to maximum ratio combining when no interference is present and the channel is known. The solid lines represent the statistic of the eight single inputs SNRs and $C / I \mathrm{~s}$. In the standard configuration of MVM (dash-dotted line) we apply $R_{c}=1^{\circ}$ and $R_{f}=0.1^{\circ}$.

TABLE IV

EFFECT OF TRACKING ON THE C/I AND SNR GAIN in SCENARIO B

\begin{tabular}{c||c|c|c|c|c}
\hline \multicolumn{1}{c||}{ DOAT configuration } & \multicolumn{5}{c}{ Scenario B } \\
\hline All values in dB & $\Delta C / I^{90 \%}$ & $\Delta C / I^{99 \%}$ & $\Delta S N R^{90 \%}$ & $\Delta S N R^{99 \%}$ & $G_{b}$ \\
\hline \hline standard & 18.5 & 18.5 & 4.5 & 3.9 & 4.3 \\
\hline no tracking & 12.9 & 0.9 & 4.8 & 3.6 & 4.5 \\
\hline theoretical optimum & - & - & 13.5 & 18.3 & 9.0 \\
\hline
\end{tabular}

We have demonstrated that DOA-based smart antenna technology can be introduced in up- and downlink of GSM systems without changes in the standard. We developed $A^{3} P$ (Adaptive Antenna Array Processor), a sophisticated system with real-time processing. We measured its uplink performance in urban mobile radio channels with actual transmitted data. As performance measures, we defined SNR and C/I gains at specific outage levels, as is required for cellular network planning.

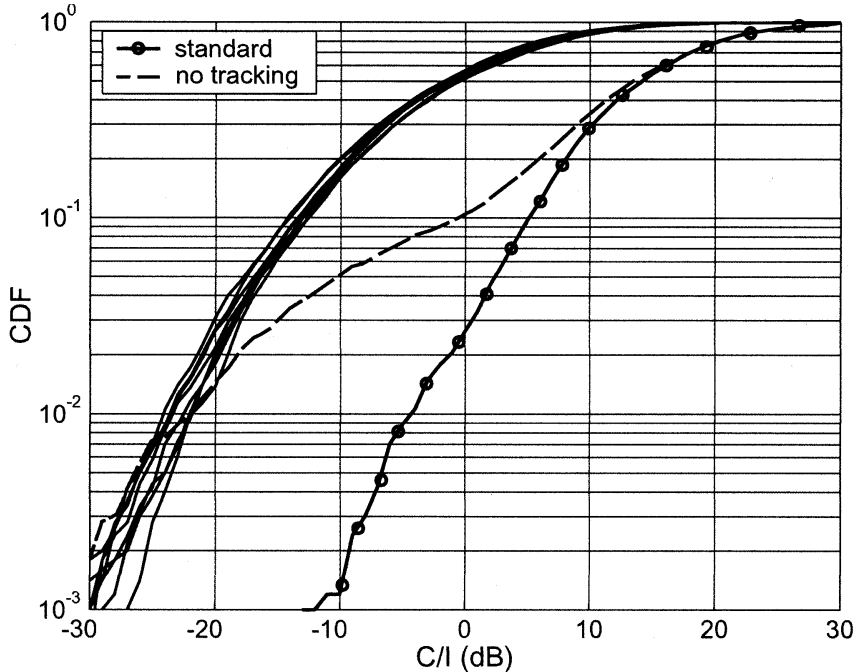

(a)

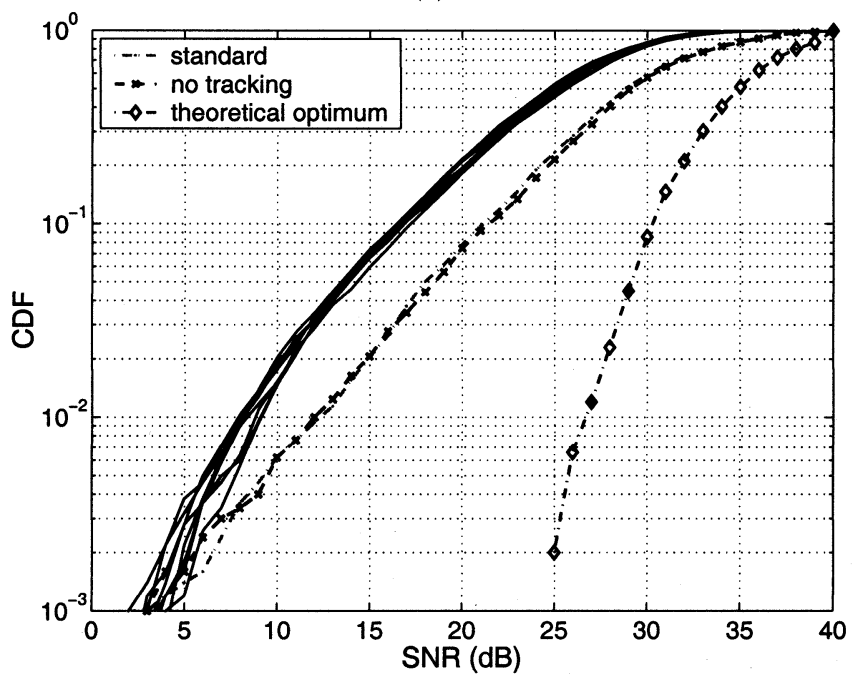

(b)

Fig. 13. Effect of the tracking concept on the $\mathrm{C} / \mathrm{I}$ and SNR statistics in Scenario B. (a) $C / I$. (b) SNR. The theoretical optimum in (b) is the sum of the single antenna element SNRs, corresponding to maximum ratio combining when no interference is present and the channel is known. The solid lines represent the statistic of the eight single inputs SNRs and C/Is.

TABLE V

ULPBF With BROAD NULLS (SMEARR) AND With CONVENTIONAL NULLS (PSEUDO INVERSE)

\begin{tabular}{c||c|c|c|c|c}
\hline \multicolumn{1}{c||}{ ULpBF configuration } & \multicolumn{5}{c}{ Scenario A } \\
\hline All values in $\mathrm{dB}$ & $\Delta C / I^{90 \%}$ & $\Delta C / I^{99 \%}$ & $\Delta S N R^{90 \%}$ & $\Delta S N R^{99 \%}$ & $G_{b}$ \\
\hline \hline broad nulls & 21.9 & 21.7 & 7.2 & 7.7 & 6.9 \\
\hline sharp nulls & 21.2 & 21.5 & 8.6 & 9.0 & 8.5 \\
\hline
\end{tabular}

The C/I gain is limited by two factors: First, the interference suppression is limited by the angular separability of user and interferer. User and interferer signals cannot be separated when all directions where the user signal can be received also include severe interference. But as long as there is a user multipath component without angular overlap, the interferer signal can be suppressed. Second, hardware imperfections limit the C/I gain if angular separability is high. 


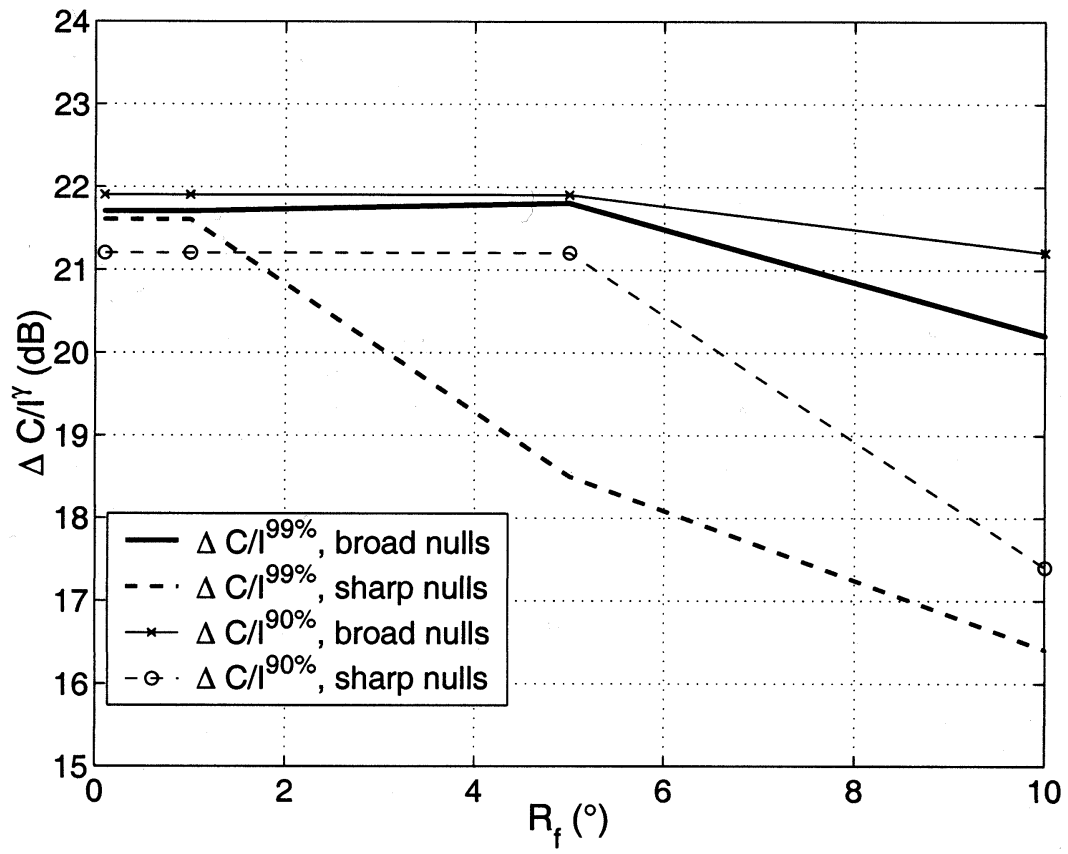

Fig. 14. Effect of broad null beamforming on the robustness of the C/I gain in Scenario A against less accurate DOA estimates. $R_{f}$ is the angular resolution of MVM. To get sharp nulls the conventional beamformer (ULpBF = Pseudo Inverse) is applied. The broad nulls are generated with SmearR.

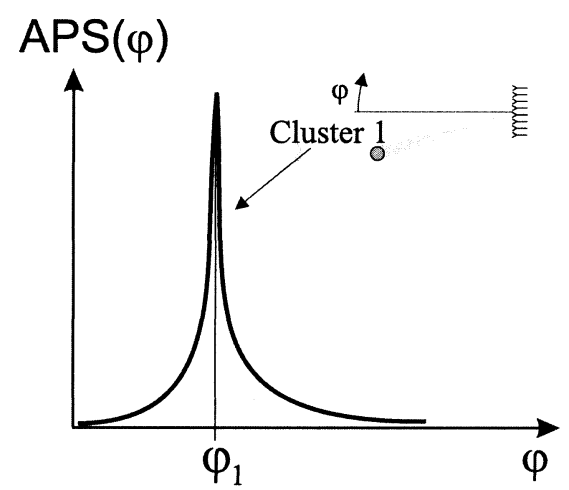

(a)

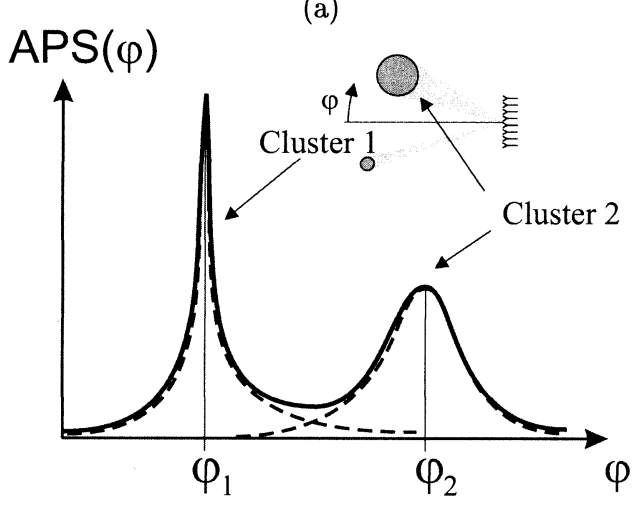

(b)

Fig. 15. Angular power spectrum, $\operatorname{APS}(\phi)$ of the user signal and corresponding channel model. (a) Single cluster and (b) two clusters, where the dashed line shows the APS of the individual clusters.

High-resolution DOA estimation with angular resolution in the subdegree range is not required, an angular resolution in the order of $5^{\circ}$ is sufficient. The relaxed requirements on the DOA estimation can be used to reduce the complexity of the system.
A variety of DOA estimators are available [11], selecting a proper one that suits the requirements of the mobile radio channel is nontrivial. We demonstrated that the estimation accuracy of a DOA estimator is not of primary concern in a cellular smart antenna application, but the robustness is. An estimator with excellent accuracy but small robustness (where far-off estimates will cause outages) is inferior to an estimator that, in every attempt, successfully estimates the DOAs, but with less accuracy. We provide an alternative indicator to assess a DOA estimator: The DOA estimation range allows assessment of the suitability of a DOA estimator in a smart antenna system. Not the averaging of the DOAs, but the concept itself guarantees high C/I gain, especially in low C/I situations. Thus, we found a compromise that allows instantaneous (burst-wise) hopping of the main beam, while reaching very high reliability at the same time.

A tracking concept that measures the signal quality of the various multipath components significantly increases the robustness against strong interference. A proper tracking concept should include features like the following.

- A main beam will only be placed in "reliable" directions. Here a direction is reliable, if the signal incident from that direction has good signal quality ver an extended period of time.

- Independent trackers to reduce the influence of far-off estimates.

- Although the tracked DOAs are averaged, the system is able to hop instantaneously from one tracked DOA to another on a burst-by-burst basis.

Concerning nulling strategy, broad nulls for the beamforming increase the robustness against poor interferer DOA estimates and against large component angular spread.

In uplink the smart antenna achieves excellent $\mathrm{C} / \mathrm{I}$ gain values up to $22 \mathrm{~dB}$. The interferer suppression is not significantly de- 
pendent on the environment. Even in an environment with large angular spread and interference from partly the same directions, the interferer suppression is as high as $18 \mathrm{~dB}$.

\section{APPENDIX I}

\section{COMPONENT ANGULAR SPREAD}

The angular spread is defined as [5]

$$
\mathrm{AS}=\sqrt{\frac{\int_{-\frac{\pi}{2}}^{\frac{\pi}{2}}(\phi-\bar{\phi})^{2} \operatorname{APS}(\phi) d \phi}{\int_{-\frac{\pi}{2}}^{\frac{\pi}{2}} \operatorname{APS}(\phi) d \phi}}
$$

where

$$
\bar{\phi}=\frac{\int_{-\frac{\pi}{2}}^{\frac{\pi}{2}} \phi \operatorname{APS}(\phi) d \phi}{\int_{-\frac{\pi}{2}}^{\frac{\pi}{2}} \operatorname{APS}(\phi) d \phi} .
$$

$\operatorname{APS}(\phi)$ is the angular power spectrum (APS) that describes the power distribution over the angle $\phi$. However, with this definition the angular spread cannot be directly applied to the performance of a DOA-based smart antenna.

The angular spread is defined as an integral over the APS that includes all signal components. Assume that we can separate the signal components of the clusters, for example, in a channel model by deactivating all clusters but one. This will result in an angular power spectrum for each cluster (see Fig. 15(b), dashed line), $\mathrm{APS}_{l}$. Thus we define the angular spread of a multipath component or in short the component angular spread (CAS), $\mathrm{CAS}_{l}$, for each cluster by applying (16) to the individual angular power spectra, $\mathrm{APS}_{l}$.

If a single cluster ${ }^{12}$ is present [see Fig. 15(a)] - or if the APS has a single peak - the angular spread and the CAS are equivalent. A large angular spread means a reduced performance of $A^{3} P$. Actually, the beamforming gain will be reduced when the CAS is larger than the main beam width, i.e., if the main beam cannot collect all offered energy. This is also true in the case of more nominal DOAs.

When more clusters (multipath components) are present [see Fig. 15(b)], the angular spread is not a good measure, but the CAS will be of importance. Just assume a situation with two well separated multipath components, each having a CAS of smaller than the main beam width. This results in a large angular spread, depending mainly by the angular separation of the multipath components. In that case $\mathrm{A}^{3} \mathrm{P}$ can better exploit the offered diversity by its angular selection diversity than in the case of a single multipath component with larger angular spread. How large the angular diversity gain of $\mathrm{A}^{3} \mathrm{P}$ will be, depends on the channel conditions. It will be optimum if more clusters are available, each with a CAS smaller than the beam width and with similar average powers. A larger CAS will again degrade the beamforming gain, but not the diversity gain.

\footnotetext{
${ }^{12}$ The term cluster is borrowed from channel modeling. In the geometrybased stochastic channel model (GSCM) [18], finite angular spread is modeled by local scatterers around the MS. These scatterers form a so-called cluster, i.e., a concentration of energy around a certain coordinate in the angle/delay domain. In GSCM typically one or two clusters are present. The second cluster is modeled by far scatterers.
}

\section{ACKNOWLEDGMENT}

The authors would like to thank C. Hoek, G. De Lattre, M. Hother, G. Köhler, and R. Weinmann for their support during the measurement campaign and G. Pospischil and M. Taferner for help in integrating the testbed.

\section{REFERENCES}

[1] F. Adachi, M. Sawashashi, and H. Suda, "W-CDMA: Performance evaluation and future enhancement (invited)," in European Personal Mobile Communications Conference (EPMCC'99), Paris, France, Mar. 1999, pp. 429-434.

[2] S. Anderson, U. Forssen, J. Karlsson, T. Witzschel, P. Fischer, and A. Krug, "Ericsson/mannesmann GSM field-trials with adaptive antennas," in IEEE Vehicular Technology Conference (VTC'97), vol. 3, Phoenix, AZ, May 1997, pp. 1587-1591.

[3] J. Capon, R. J. Greenfield, and R. J. Kolker, "Multidimensional maximum-likelihood processing of a large aperture seismic array," in Proc. IEEE, vol. 55, Feb. 1967 , pp. 192-211.

[4] C. K. Chui and G. Chen, Kalman Filtering With Real-Time Applications. New York: Springer-Verlag, 1991.

[5] P. C. F. Eggers, "Angular dispersive mobile radio environments sensed by highly directive base station antennas," in International Symposium on Personal, Indoor and Mobile Radio Communication (PIMRC'95), vol. 1, Toronto, Canada, Sept. 1995, pp. 522-526.

[6] U. Forssen, J. Karlsson, B. Johannison, F. Kronstedt, F. Lotse, M. Almgren, and S. Anderson, "Adaptive antenna arrays in GSM900/DCS1800," in IEEE Vehicular Technology Conference (VTC'94), Stockholm, Sweden, June 1994, pp. 605-609.

[7] M. Haardt and J. A. Nossek, "Unitary ESPRIT: How to obtain increased estimation accuracy with a reduced computational burden," IEEE Trans. Signal Processing, vol. 43, no. 5, pp. 1232-1242, May 1995.

[8] B. Hagerman, T. Östman, K. J. Molnar, and G. E. Bottomley, "Field test performance results for D-AMPS in PCS bands with array processing," in IEEE Vehicular Technology Conference (VTC'97), vol. 3, Phoenix, AZ, May 1997, pp. 1582-1586.

[9] W. C. Jakes, Microwave Mobile Communications. New York: IEEE Press, 1974.

[10] D. H. Johnson and D. E. Dudgeon, Array Signal Processing, Concepts and Techniques: Prentice-Hall Signal Processing Series, 1991.

[11] H. Krim and M. Viberg, "Two decades of array signal processing research," IEEE Signal Processing Magazine (Special Issue on Array Processing), vol. 13, pp. 67-94, Feb. 1996.

[12] A. Kuchar, "Real-Time Smart Antenna Processing for GSM1800," Ph.D. Thesis, Technische Universität Wien, Feb. 2000.

[13] A. Kuchar, M. Taferner, M. Tangemann, C. Hoek, W. Rauscher, M. Strasser, G. Pospischil, and E. Bonek, "Real-time smart antenna processing for GSM1800 base station," in IEEE Vehicular Technology Conference (VTC'99), Houston, TX, May 1999, pp. 664-669.

[14] — , "A robust DOA-based smart antenna processor for GSM base stations," in IEEE International Conference on Communications (ICC'99), Vancouver, BC, Canada, June 1999, pp. 11-16.

[15] J. Laurila, A. F. Molisch, and E. Bonek, "Influence of the scatterer distribution on power delay profiles and azimuthal power spectra of mobile radio," in IEEE International Symposium on Spread Spectrum Techniques and Applications (ISSSTA'98), Sun City, South Africa, Sept. 1998, pp. 267-271.

[16] P. Mogensen, F. Frederiksen, H. Dam, K. Olesen, and S. L. Larsen, "TSUNAMI (II) stand alone testbed," in ACTS Summit'96, vol. 1, Granada, Spain, Nov. 1996, pp. 517-527.

[17] P. Mogensen, K. Pedersen, P. Leth-Espensen, B. Fleury, F. Frederiksen, K. Olesen, and S. Larsen, "Preliminary measurement results from an adaptive antenna array testbed for GSM/UMTS," presented at the IEEE Vehicular Technology Conference (VTC'97), Phoenix, AZ, May 1997.

[18] A. F. Molisch, A. Kuchar, J. Laurila, K. Hugl, and E. Bonek, "Efficient implementation of a geometry-based directional model for mobile radio channels," in IEEE Vehicular Technology Conference (VTC'99 Fall), Amsterdam, The Netherlands, Sept. 1999, pp. 1449-1453.

[19] H. Novak, H. Nord, and A. Kuchar, "A single-layer $8 \times 8$ butler matrix with patch antenna," in MTT-S European Wireless'98, Amsterdam, The Netherlands, Oct. 1998, pp. 25-29.

[20] M. Taferner, A. Kuchar, M. C. Lang, M. Tangemann, and C. Hoek, "A novel DOA-based beamforming algorithm with broad nulls," presented at the International Symposium on Personal, Indoor and Mobile Radio Communication (PIMRC'99), Osaka, Japan, Sept. 1999. 
[21] M. Tangemann, C. Hoek, and R. Rheinschmitt, "Introducing adaptive array antenna concepts in mobile communication systems," in Proc. RACE Mobile Communications Workshop, Amsterdam, The Netherlands, May 1994, pp. 714-727.

[22] M. Wax and T. Kailath, "Detection of signals by information theoretic criteria," IEEE Trans. Acoust., Speech, and Signal Processing, vol. 33, no. 2, Apr. 1985.

[23] J. H. Winters, "Optimum combining in digital mobile radio with cochannel interference," IEEE J. Select. Areas Commun., vol. 2, no. 4 pp. 528-539, July 1984.

[24] J. H. Winters, C. C. Martin, and T. Zhang, "A two-element adaptive antenna array for IS-136 PCS base stations," presented at the Proc. USCD Conference, Mar. 1998.

[25] B. Yang, "Projection approximation subspace tracking," IEEE Trans. Signal Processing, vol. 43, pp. 95-107, Jan. 1995.

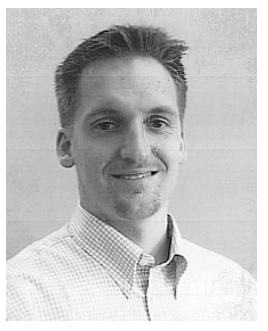

Alexander Kuchar (S'96-M'01) was born in Vienna, Austria, in 1971. He received the Dipl.-Ing. and Dr.techn. degrees (with highest honors) in electrical engineering from the Technische Universität Wien (TU Wien), Vienna, Austria, in 1996 and 2000, respectively.

From 1995 to 2000, he was a member of the Mobile Communciations Group at the Institut für Nachrichtentechnik und Hochfrequenztechnik of Technische Universität Wien, where he lead the $A^{3} P$ project. During that time, his main research interests were in signal processing for smart antennas for mobile communication systems, directional channel modeling, and microstrip antenna arrays. Since mid-2000, he has been with Mobilkom Austria, where he is now working on UMTS service architectures.

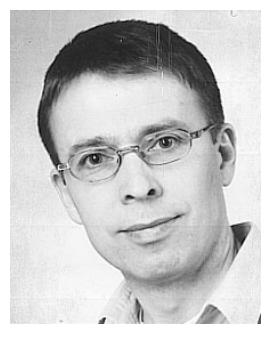

Michael Tangemann (M'94) was born in Gummerbach, Germany, in 1961. He received the Dipl.-Ing. and Dr.-Ing. degrees in electrical engineering from the University of Stuttgart, Germany, in 1987 and 1994, respectively.

From 1987 to 1992 he worked at the Institute of Communication Networks and Computer Engineering, University of Stuttgart, in the area of High-Speed Local Area Network architecture and performance evaluation. After joining Alcatel Research and Innovation in 1992, he worked in several research projects focussing on the application of smart antenna technology in mobile communication systems. Since 1999, he has been involved in various projects dealing with packet-switched data transfer in mobile networks, including EDGE and issues of wireless TCP transfers.

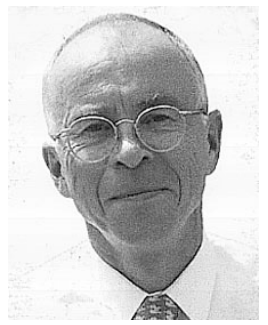

Ernst Bonek (M'73-SM'85) was born in Vienna, Austria, 1942. He received the Dipl.-Ing. and Dr.techn. degrees (with highest honors) from the Technische Universität Wien (TU Wien), Vienna, Austria.

In 1984, he was appointed Full Professor of Radio Frequency Engineering at the TU Wien. His field of interest is mobile communications at large. His recent contributions concern smart antennas, the characterization of mobile radio channels including MIMO and mobile networks. He is coauthor of Wireless internet access over GSM and UMTS (New York: Springer). He has authored or coauthored about 150 journal and conference publications and holds several patents on mobile radio technology.

Dr. Bonek has served as a Chairman for the IEEE Austria Section from 1985 to 1990 . From 1991 to 1994 , he was a council member of the Austrian Science Fund, acting as speaker for engineering sciences. From 1996 to 1999, he served on the Board of Directors of the reorganized Post and Telekom Austria. He participated in the European research initiative COST 259 as Chairman of the Working Group on Antennas and Propagation, and continues to serve in this position in COST 273. In URSI, he was Chairman of Commission C "Signals and Systems" between 1999 and 2002. He is an Area Editor of Wireless Personal Communications by Kluwer. 